Mitteilungen der Österreichischen Geographischen Gesellschaft, 162. Jg., S. 255-285

(Annals of the Austrian Geographical Society, Vol. 162, pp. 255-285)

Wien (Vienna) 2020, https://doi.org/10.1553/moegg162s255

\title{
KULTURLANDSCHAFTSFORSCHUNG
}

CULTURAL LANDSCAPE RESEARCH

\section{KULTURLANDSCHAFT IM WANDEL: EIN INDIKATOREN- \\ BASIERTER RÜCKBLICK BIS IN DAS 19. JAHRHUNDERT \\ Fallstudie anhand der Gemeinden Waidhofen/Ybbs und Paldau}

\author{
Raphael Knevels (Jena), Alexander BrenNING (Jena), Simone GingRICH, \\ Elisabeth GRUBER, Theresia LECHNER, Philip LEOPOLD (alle Wien), \\ Helene PetschKo (Jena), Christoph Plutzar (Wien)*
}

Erste Einreichung / initial submission: 05/2020; revidierte Fassung / revised submission: 12/2020; endgültige Annahme / final acceptance: 12/2020

mit 4 Abb. und 8 Tab. im Text

\section{INHALT}

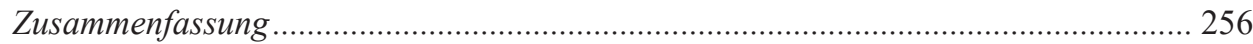

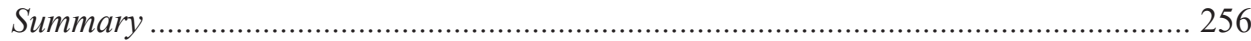

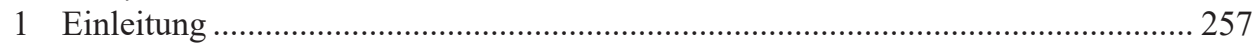

2 Untersuchungsgebiet, Datenerhebung und kontextuelle Einordnung ..................... 259

3 Quantifizierung des Kulturlandschaftswandels ........................................................ 263

* Raphael Knevels M.Sc., Friedrich-Schiller-Universität Jena, Institut für Geographie, Grietgasse 6, D-07743 Jena, https://orcid.org/0000-0002-5094-7206; - Prof. Dr. Alexander BRENNING, Friedrich-Schiller-Universität Jena, Institut für Geographie, Grietgasse 6, D-07743 Jena, https://orcid.org/0000-0001-6640-679X; - Dr. Simone GINGRICH, Universität für Bodenkultur Wien, Institut für Soziale Ökologie, Schottenfeldgasse 29, A-1070 Wien, https://orcid.org/0000-0001-7891-8688; - Mag. Dr. Elisabeth GruBER, Österreichische Akademie der Wissenschaften, Institut für Stadt- und Regionalforschung, Vordere Zollamtsstraße 3, A-1030 Wien; - Dipl.Ing. Theresia LEChNER, Universität für Bodenkultur Wien, Institut für Soziale Ökologie, Schottenfeldgasse 29, A-1070 Wien; - Dr. Philip Leopold, AIT Austrian Institute of Technology GmbH, Center for Mobility Systems, Giefinggasse 2, A-1210 Wien; - Mag. Dr. Helene Petschкo, Friedrich-Schiller-Universität Jena, Institut für Geographie, Lehrstuhl für Geoinformatik, Grietgasse 6, D-07743 Jena, https://orcid.org/0000-0001-9712-4859; - Dr. Christoph Plutzar, Universität für Bodenkultur Wien, Institut für Soziale Ökologie, Schottenfeldgasse 29, A-1070 Wien; Universität Wien, Division of Conservation Biology, Vegetation Ecology and Landscape Ecology, Rennweg 14, A-1030 Wien, https://orcid.org/0000-0003-2041-6399. - E-Mails: raphael.knevels@unijena.de, alexander.brenning@uni-jena.de, simone.gingrich@boku.ac.at, elisabeth.gruber@oeaw.ac.at, philip. leopold@ait.ac.at, helene.petschko@uni-jena.de, christoph.plutzar@univie.ac.at. 


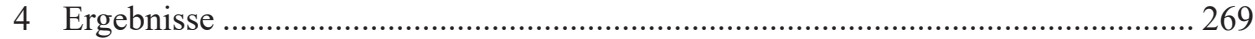

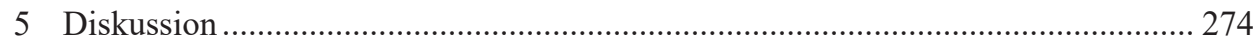

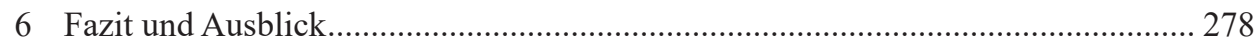

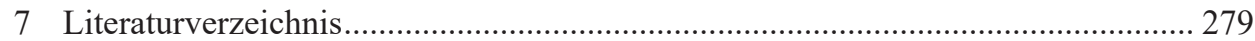

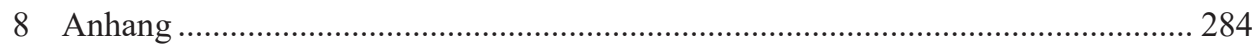

\section{Zusammenfassung}

Die Nutzung und die Umgestaltung von Landschaften haben sich seit Beginn der Industrialisierung stark verändert. Um die Flächenentwicklung zu erfassen und hinsichtlich nachhaltiger Raumentwicklung regelmäßig zu beobachten, braucht es geeignete Mittel. Indikatorensysteme helfen, diesen Anforderungen gerecht zu werden. Es gibt bereits zahlreiche indikatorenbasierte Informations- und Bewertungsinstrumente, die die Flächenentwicklung unter quantitativen wie qualitativen Gesichtspunkten bewerten. Diesbezügliche Untersuchungen decken allerdings aufgrund der Datenbasis häufig nur einen rezenten Zeitraum ab. Die Identifikation von auf langen historischen Zeiträumen basierenden Trends bietet die Möglichkeit, die aktuelle Situation umfassender zu kontextualisieren.

In dieser Arbeit analysieren wir die Kulturlandschaftsentwicklung in zwei österreichischen Gemeinden im Voralpenraumn bzw. im Südöstlichen Alpenvorland über einen Zeitraum von nahezu 200 Jahren. Dazu wurden aus Katasterkarten, Luftbildern und Orthophotos konsistente, räumlich explizite Landnutzungsdatensätze für drei Zeitschnitte (1820, 1960, 2015) erstellt. Der Fokus liegt auf der Siedlungs-, Wald- und Landwirtschaftsentwicklung, für die raumbezogene Indikatoren aus geeigneten Indikatorensystemen zur Beobachtung nachhaltiger Raumentwicklung ausgewählt wurden. Für jeden Zeitschnitt wurden die Indikatoren zu einem Gesamtindex des Kulturlandschaftswandels aggregiert und anschließend verglichen. Das verwendete Indikatorensystem eignet sich, um langfristige Trends anhand von Flächenentwicklungen darzustellen. In beiden untersuchten Gemeinden ist der Gesamtindex heute deutlich geringer als um 1820, was eine negativ gewertete Kulturlandschaftsentwicklung darstellt. Obwohl Unterschiede in den betrachteten Entwicklungspfaden bestehen, weist die Siedlungsentwicklung im Vergleich zur Wald- und zur Landwirtschaftsentwicklung in beiden Gemeinden den stärksten negativen Trend auf.

Schlagwörter: Kulturlandschaftswandel, Indikatoren, Landschaftsstrukturmaße, freie Programmierumgebung $R$, Indikatorensystem, Alpenraum, Voralpen, Alpenvorland, Niederösterreich, Steiermark

\section{Summary}

\section{Cultural Landscape Change: An Indicator-Based Retrospect into the $19^{\text {th }}$ Century. Case Study of the Municipalities Waidhofen/YbBS AND PALDAU}

During the industrialisation the use of land has changed considerably. Appropriate tools are needed to monitor land use trends and sustainable development. These requirements 
are met by indicator systems. Indicator-based frameworks are available for the assessment of land development from both, quantitative and qualitative points of view. However, these studies are usually limited to a recent period due to data availability. Analysing long-term trends offers the possibility of apprehending the current situation in a broader context.

In this paper, we analysed the cultural landscape change of two Austrian municipalities in the alpine fringe region covering almost 200 years. We created spatially explicit land use data for three time cuts $(1820,1960,2015)$ based on historical cadastral maps, aerial photographs and orthophotos. Our analysis focused on the development paths of settlement, forest and agricultural areas, for which geospatial indicators of sustainable development were selected. For each time cut, the indicators were aggregated to an overall index and then compared. The implemented indicator system was suitable to detect trends in land use changes. In both study areas the overall index is significantly lower today than it was in 1820, which represents a negatively valued cultural landscape change. Although there are differences in the development paths, settlement development shows the strongest negative trend in both communities.

Keywords: Cultural landscape change, indicators, landscape metrics, $R$ programming, indicator system, Alpine region, Pre-Alps, Alpine Foreland, Lower Austria, Styria

\section{Einleitung}

Die Nutzung und die Umgestaltung von Landschaften haben sich seit Beginn der Industrialisierung stark verändert. Die Kulturlandschaft, verstanden als die vom Menschen nach gesellschaftlichen, wirtschaftlichen und kulturell-ästhetischen Bedürfnissen überprägte Erdoberfläche (SCHENK 2016, S. 19), ist stetig im Wandel. WALZ und STEIN (2017, S. 58) benennen vier wesentliche Prozesse, die aktuell zum mitteleuropäischen Kulturlandschaftswandel beitragen: 1. die Neuinanspruchnahme von Flächen durch Siedlung und Verkehr, 2. die Zerschneidung durch Infrastruktur, 3. die Nutzungsintensivierung in der Landwirtschaft, und 4. der flächenintensive Ausbau erneuerbarer Energien. Während der Ausbau erneuerbarer Energien ein relativ rezentes Phänomen darstellt (z. B. Freiflächen-Solaranlagen, Windkraftanlagen und Bioenergie, WALZ und STEIN 2017, S. 64), prägen die anderen Prozesse seit dem 18. Jahrhundert mit einer zunehmenden Intensivierung bedeutend das Landschaftsbild (HAMPICKE 2013).

Etwa die Hälfte der Landschaften Österreichs sind Kulturlandschaften mit höchster oder hoher Schutzwürdigkeit (WrBKA et al. 2005). Nach WrBKA et al. (2005, S. 44-52) leitet sich die Schutzwürdigkeit aus naturschutzfachlichen Bewertungskriterien wie dem Alter bzw. der Persistenz der Landschaft, dem menschlichen Kultureinfluss (Hemerobie), dem Anteil an Kleinstrukturen oder der biologischen Vielfalt ab (Diversitäts-Hot-Spots, Anteil an „Important Bird Areas“). So sind Kulturlandschaften mit höchster oder hoher Schutzwürdigkeit vor allem Natur- oder naturnahe Landschaften, aber auch traditionell genutzte Landschaften, die eine hohe Lebensraumvielfalt aufweisen (ebd., S. 9). Zwar werden Kulturlandschaften seit den 1990er Jahren in Österreich gesetzlich als besonders schützens- und erhaltenswert erachtet (BGB1. 1992), jedoch ist der Großteil der Land- 
schaften höchster Schutzwürdigkeit ohne Schutzstatus (über $60 \%$, WRBKA et al. 2005, S. 92). Der Alpenraum stellt hierbei mit seiner sehr großen Artenvielfalt und seinen hoch-empfindlichen Biozönosen eine besonders schützenswerte Landschaft im mitteleuropäischen Kontext dar (BÄTZING 2017; ScHINDLER et al. 2017), was auch im Rahmen der Alpenkonvention Ausdruck findet (BGB1. 1995).

Der Schutz und Erhalt der Kulturlandschaft ist zudem Bestandteil nationaler wie internationaler Nachhaltigkeitsstrategien und -ziele (z. B. ÖREK, EUREK; vgl. KLEINER 2006) und hat somit deren raumordnerische Bedeutung erhöht. Über eine stetige Raumbeobachtung können meist schleichend verlaufende, strukturelle Veränderungen der Kulturlandschaft erkennbar gemacht werden (WALZ 2013). Allerdings gibt es erst seit wenigen Jahrzehnten ein aktives Monitoring, sodass die Evaluierung von Nutzungsveränderungen mit ihren Auswirkungen zeitlich begrenzt ist. Fragen zum Kulturlandschaftswandel sind zudem meist zukunftsgerichtet: So bieten Landnutzungsszenarien die Möglichkeit, Raumplanungsmaßnahmen und ihre Auswirkungen auf unterschiedliche Aspekte flächenpolitischer Ziele oder Leitbilder hin zu untersuchen (z. B. Beitrag zur kompakten Innenentwicklung oder Flächenneuinanspruchnahme; vgl. HoymanN und GoETZKE 2014; 2016).

Die Landschaftsgeschichte liefert jedoch in dem Vermächtnis von in der Vergangenheit geschaffenen, aber noch bestehenden Formen (z. B. Städte, Denkmäler) oder Spuren (z. B. Ruinen, Wüstungen) das grundlegende Verständnis gegenwärtiger Strukturen für die Planung und Raumwissenschaft (BENDER 2007, S. 31-32; „Landschaft als Archiv der Vergangenheit“; SCHENK 2002, S. 55). Auch kann sie als „historisches Leitbild“ eine Vorbildfunktion für künftige Entwicklungen einnehmen oder ein Lernen für die Zukunft ermöglichen (z. B. über Landnutzungsszenarien oder -management; BENDER 2003, S. 137; MARCUCCI 2000, S. 76-79).

Um die ,jüngere“ Kulturlandschaftsentwicklung und somit den Übergang von der vorindustriellen, traditionellen Agrargesellschaft hin zur dienstleistungsorientierten Gesellschaft zu erfassen, empfiehlt BENDER (2007, S. 68), einen Untersuchungszeitraum von 200 Jahren zu wählen (vgl. BENDER et al. 2005; TASSER et al. 2009; WALz 2008). Aufgrund der limitierenden Datenverfügbarkeit verlässlicher Quellen (z. B. Historische, Topographische und Katasterkarten), verwenden einige Untersuchungen jedoch Fernerkundungsdaten und bleiben somit zeitlich bis zur Mitte des 20. Jahrhunderts beschränkt (z. B. OLSSON et al. 2000; SALVATI et al. 2017).

Qualitativ beschrieb BäTZING (2002; 2017) den Kulturlandschaftswandel im alpinen Raum seit der Industrialisierung und identifizierte drei kennzeichnende Phasen: 1. Phase der Industriegesellschaft (1880-1950), 2. Phase der Übergangsgesellschaft (1950-1980), und 3. Phase der Dienstleistungsgesellschaft (seit 1980). In einer quantitativen Untersuchung für neun Untersuchungsgebiete in Südtirol beschrieben TASSER et al. (2009) den Landnutzungswandel von 1865 bis 2000 (weitere Zeitschnitte 1955 und 1980) mithilfe von vier Indikatoren (Landnutzungsart, Habitatkonfiguration, Strukturierungsgrad der Landschaft und Zersiedlung). Sie erkannten u. a. einen generellen Trend zur monotonen Landschaftsstruktur, eine signifikante Abnahme offener Kulturlandschaften und eine Vervierfachung der Siedlungsfläche (ebd., S. 619-620).

Weitere quantitative Untersuchungen für einen längeren Zeitraum fokussierten vor allem auf die Landschaftszerschneidung, gemessen mithilfe des Indikators der „effektiven 
Maschenweite“ als Messgröße unzerschnittener Flächen (JAEGER 2000). So untersuchten BERTILLER et al. (2007) die Landschaftszerschneidung der Schweiz im Zeitraum von 1885 bis 2002 anhand von fünf Zeitschnitten (1885, 1935, 1960, 1980 und 2002). Sie stellten fest, dass seit 1885 die effektive Maschenweite um 56 bis 70 Prozent abgenommen hat. Vergleichbare Untersuchungen zeigten ähnliche Trends (z. B. Hessen für den Zeitraum 1930 bis 2002, RoEDENBECK 2005; Baden-Württemberg für den Zeitraum 1930 bis 1998 , EssweIN et al. 2002). Andere räumlich explizite Langzeitstudien zum Landnutzungswandel fokussierten auf sozio-ökologische Veränderungen wie die Eingriffe in den Kohlenstoffhaushalt von Ökosystemen (NIEDERTSCHEIDER et al. 2017) oder die Auswirkungen auf Habitatveränderungen (PARCERISAS et al. 2012).

In diesem Beitrag haben wir das Ziel, den flächenbezogenen Kulturlandschaftswandel zweier österreichischer Gemeinden über einen Zeitraum von nahezu 200 Jahren zu analysieren. Der Fokus unserer Analyse geht hierbei über eine reine Betrachtung von Flächenstatistiken hinaus und liegt auf der Erfassung struktureller Veränderungen des Landnutzungsmusters. Hierfür quantifizieren wir in Anlehnung an WALZ und STEIN (2017) drei Entwicklungspfade zur Siedlungs-, Wald-, und Landwirtschaftsentwicklung und evaluieren diese mithilfe eines von uns entwickelten Indikatorensystems. Bisher gibt es kein einheitliches Set an Indikatoren zum Monitoring des Kulturlandschaftswandels (WALz 2013). Die Basis unseres Indikatorensystems stellt eine Auswahl an Indikatoren dar, die zur Beschreibung rezenter flächenpolitischer Ziele und Leitbilder einer nachhaltigen Raumentwicklung entwickelt wurden und auf historische Daten anwendbar sind (SIEDENTOP et al. 2007; WALZ und STEIN 2017). Die Landnutzung im Untersuchungszeitraum wird anhand räumlich expliziter Datensätze für drei Zeitschnitte (1820, 1960, 2015) abgebildet. Die Untersuchungsgebiete befinden sich im (Vor)Alpenraum gemäß der Abgrenzung durch die Alpenkonvention (BÄTZING 2001; BGBl. 1995). Dies ermöglicht eine Einordnung identifizierter Tendenzen in BäTZINGs (2002; 2017) kennzeichnende Phasen des Kulturlandschaftswandels in den Alpen.

\section{Untersuchungsgebiet, Datenerhebung und kontextuelle Einordnung}

Unsere Untersuchung fand in zwei Gemeinden Österreichs statt: in der niederösterreichischen Gemeinde Waidhofen an der Ybbs (im Folgenden Waidhofen) und in der steirischen Gemeinde Paldau. Beide Gemeinden repräsentieren unterschiedliche Naturräume, die auch unterschiedlichen Landschaftsveränderungen unterliegen (Abb. 1). Waidhofen liegt in den Ybbstaler Alpen, umfasst eine Fläche von $131 \mathrm{~km}^{2}$ und hat heute etwa 13.000 Einwohner (Statistik Austria 2011a). Die Gemeinde weist einen Nord-Süd-Gradienten auf: im Kalkstein-dominierten Süden werden bis zu 1.206 m Höhe über dem Meeresspiegel (m ü. Adria) erreicht, zur Flysch-Zone im Norden der Gemeinde flachen die Berge ab (302 m ü. Adria; Wessely 2006). Im Gegensatz dazu liegt Paldau im Oststeirischen Becken (charakterisiert durch Lockersedimente des Neogens bis Quartärs; GROss 2003, S. 29-31) - eine landwirtschaftliche Gunstlage, die heute von Mais- und Schweineproduktion geprägt ist. Die Gemeinde erstreckt sich über $39 \mathrm{~km}^{2}$, und heute leben dort etwas mehr als 3.000 Einwohner (Statistik Austria 2011b; 282 bis 465 m ü. Adria). 


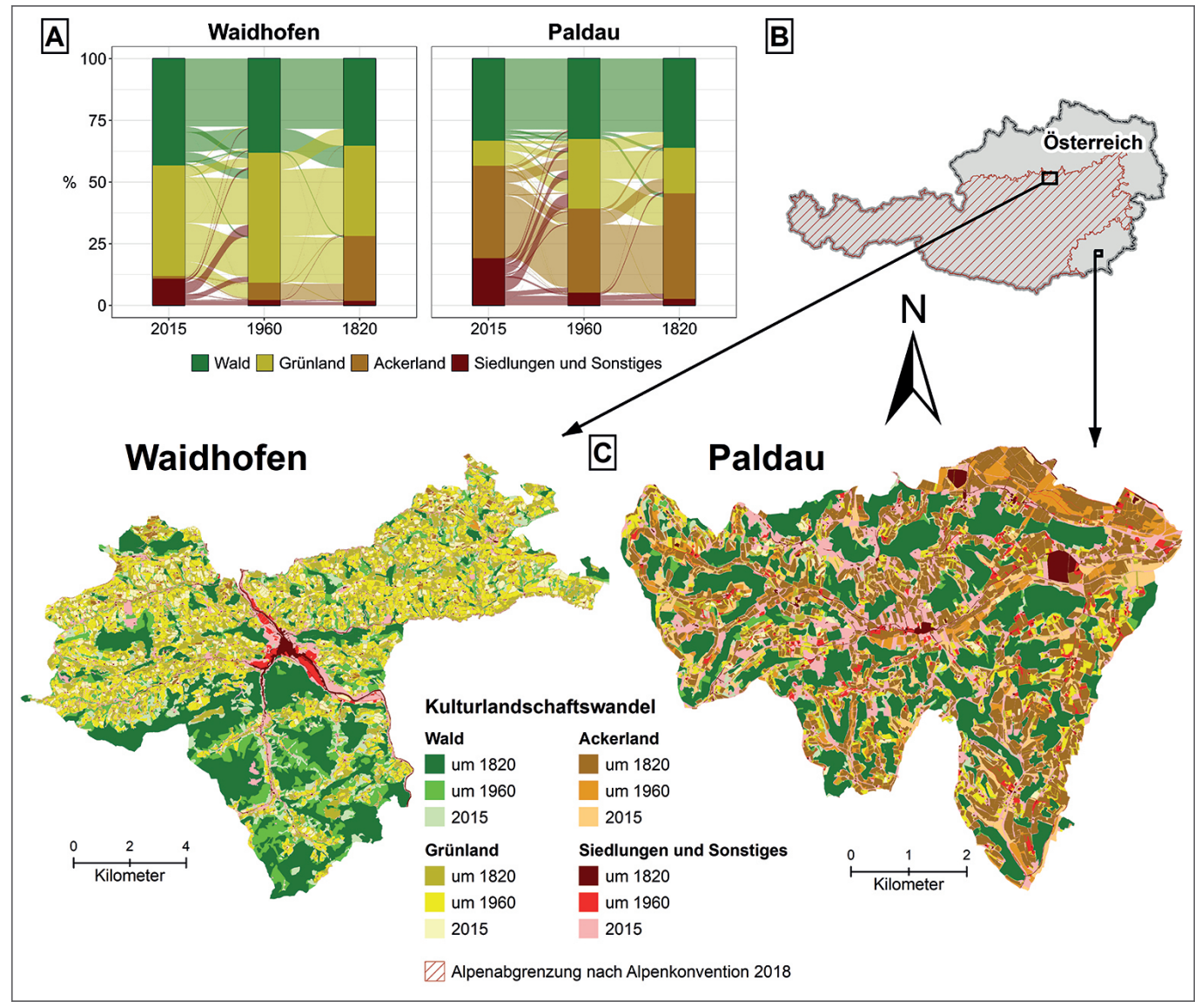

A: Anteil und Veränderung der Landnutzungsklassen; B: Verortung in Österreich; C: Kulturlandschaftswandelkarte nach Landnutzungsklassen für Waidhofen (links) und Paldau (rechts).

Quelle: Eigene Darstellung

Abb. 1: Kulturlandschaftswandel im Untersuchungsgebiet und -zeitraum

Die Auswahl der Untersuchungsgebiete fand im Rahmen eines Forschungsprojekts statt (siehe ILLAS 2020): Beide Untersuchungsgebiete zeigen trotz ihres unterschiedlichen Reliefs eine hohe Prädisposition für Hangrutschungen. Die Analyse der Kulturlandschaftsentwicklung kann helfen, den Zusammenhang zwischen solchen Naturgefahren und Landnutzungsänderungen besser zu erklären (vgl. REICHENBACH et al. 2014).

Für beide Gemeinden wurde auf Basis von Primärdaten eine Geodatenbank erstellt, die Informationen zu Landnutzung und Landbedeckung für drei Zeitschnitte (1820, 1960 und 2015) konsistent ausweist (Tab. 1). Die einem Zeitschnitt zugeordneten Datenquellen können in ihrem Entstehungsjahr zwischen den Gemeinden abweichen. Die Wahl der Zeitschnitte ergab sich aus der Datenverfügbarkeit (zeitliche Nähe der Statistiken und 
räumlichen Informationen) und orientierte sich an den Empfehlungen von BENDER (2007, S. 68-69), der Zeitschnitte basierend auf sozioökonomischen Rahmenbedingungen für die mitteleuropäische Kulturlandlandschaft ableitet (d. h., um 1850, 1914, 1960 sowie einen aktuellen Zeitschnitt). Auch haben sich die gewählten Zeitschnitte in Studien mit vergleichbarer Datenlage bewährt (vgl. BENDER et al. 2005; TASSER et al. 2009).

\begin{tabular}{|c|c|c|c|c|c|}
\hline Jahr & $\begin{array}{l}\text { Fall- } \\
\text { studie }\end{array}$ & Quelle & $\begin{array}{c}\text { Quellen- } \\
\text { verfügbarkeit }\end{array}$ & Qm & Begründung \\
\hline \multicolumn{6}{|c|}{ Datentyp: Landnutzung } \\
\hline \multirow{2}{*}{1820} & Wh & \multirow{2}{*}{$\begin{array}{l}\text { Urmappe des } \\
\text { Franziszeischen } \\
\text { Katasters }\end{array}$} & $\begin{array}{l}\text { Niederösterreichisches } \\
\text { Landesarchiv }\end{array}$ & ++ & \multirow{2}{*}{$\begin{array}{l}\text { Scharfe Abgrenzung der } \\
\text { Nutzungseinheit }\end{array}$} \\
\hline & $\mathrm{P}$ & & $\begin{array}{l}\text { Bundesamt für Eich- und } \\
\text { Vermessungswesen }\end{array}$ & ++ & \\
\hline 1962 & $\mathrm{Wh}$ & \multirow{2}{*}{ Luftbilder } & \multirow{2}{*}{$\begin{array}{l}\text { Bundesamt für Eich- und } \\
\text { Vermessungswesen }\end{array}$} & $\sim$ & \multirow{2}{*}{$\begin{array}{l}\text { Abgrenzung basierend auf } \\
\text { Schwarz-Weiß-Luftbildern }\end{array}$} \\
\hline 1953 & $\mathrm{P}$ & & & $\sim$ & \\
\hline 2015 & Wh, P & $\begin{array}{l}\text { Orthophotos und } \\
\text { InVeKoS* }\end{array}$ & $\begin{array}{c}\text { Open Government Data } \\
\text { Licence }\end{array}$ & ++ & $\begin{array}{l}\text { Parzellenscharfe Abgrenzung } \\
\text { von Acker- und Grünland }\end{array}$ \\
\hline \multicolumn{6}{|c|}{ Datentyp: Landwirtschaftliche Erträge (Getreide- und Wiesenertrag) } \\
\hline 1820 & Wh & $\begin{array}{c}\text { Franziszeische } \\
\text { Kataster Elaborate }\end{array}$ & $\begin{array}{c}\text { Niederösterreichisches } \\
\text { Landesarchiv }\end{array}$ & + & $\begin{array}{l}\text { Daten für zwei der Katastral- } \\
\text { gemeinden von Waidhofen }\end{array}$ \\
\hline 1820 & $\mathrm{P}$ & SANDGRUBER 1978 & & $\sim$ & Durchschnittswert für Steiermark \\
\hline 1960 & Wh, P & Agrarstatistik & $\begin{array}{l}\text { Bibliothek der } \\
\text { Statistik Austria }\end{array}$ & ++ & Daten auf Gemeindeebene \\
\hline 2015 & Wh, P & InVeKoS* & data.gv.at & + & Daten für Betriebe in Gemeinden \\
\hline \multicolumn{6}{|c|}{ Datentyp: Holzertrag } \\
\hline 1820 & Wh & $\begin{array}{c}\text { Franziszeischer } \\
\text { Kataster Elaborate }\end{array}$ & $\begin{array}{l}\text { Niederösterreichisches } \\
\text { Landesarchiv }\end{array}$ & + & $\begin{array}{l}\text { Daten für zwei der Katastralge- } \\
\text { meinden von Waidhofen }\end{array}$ \\
\hline 1820 & $\mathrm{P}$ & GINGRICH et al. 2007 & & $\sim$ & $\begin{array}{l}\text { Durchschnittswert für } \\
\text { Steiermark }\end{array}$ \\
\hline 1965 & Wh, P & WeIss et al. 2000 & & $\sim$ & $\begin{array}{l}\text { Durchschnittswert für } \\
\text { Österreich }\end{array}$ \\
\hline 2015 & Wh, P & Forstinventur & bfw.gv.at & $\sim$ & $\begin{array}{l}\text { Durchschnittswert für } \\
\text { Bundesländer }\end{array}$ \\
\hline \multicolumn{6}{|c|}{ Datentyp: Bevölkerung } \\
\hline $\begin{array}{l}1869 \\
1960 \\
2015\end{array}$ & Wh, P & $\begin{array}{l}\text { Statistik Austria } \\
\text { 2011a; 2011b }\end{array}$ & statistik.at & + & $\begin{array}{l}\text { für } 1820 \text { wurde der Wert von } \\
1869 \text { angenommen }\end{array}$ \\
\hline
\end{tabular}

Wh: Waidhofen, P: Paldau; - * Datenstand der zeitlichen Ausdehnung von 2016; - Qm (Qualitätsmaße) +++ .. räumliche und inhaltliche Qualität gut; + ... räumliche oder inhaltliche Qualität gut; ... räumliche und inhaltliche Qualität ausreichend

Tab. 1: Verwendete Quellen zur Erstellung der Geodatenbank und Einschätzung ihrer Qualität nach Datentyp 
Aus den räumlich expliziten Quellen wurden vier Landnutzungskategorien für alle drei Zeitschnitte abgeleitet: Ackerland, Grünland, Wald sowie Siedlungen und Sonstiges. Ackerland und Grünland stellen die landwirtschaftlichen Flächen dar.

Die Heterogenität der Primärdaten machte es notwendig, besonderes Augenmerk auf Konsistenz während der Erfassung zu legen: Alle Karten wurden von derselben Person (T. LECHNER) nach einheitlichen Kriterien jeweils für eine Untersuchungsregion on-screen digitalisiert. Während der Digitalisierung wurde durchgehend eine Maßstabsdarstellung von 1:1000 verwendet. Die Reihenfolge der Digitalisierung ergab sich aus der vorhandenen Flächenschärfe der Primärdaten. Zunächst wurden die flächenscharfen Kartenblätter des Franziszeischen Katasters (Zeitschnitt 1820) georeferenziert und erfasst (Tab. 2). Für den Zeitschnitt 2015 standen flächenscharfe InVeKoS-Daten (InVeKoS: Integriertes Verwaltungs- und Kontrollsystem) und Orthophotos zur Verfügung. Aus InVeKoS wurden rezente Acker- und Grünlandflächen übernommen und visuell überprüft (AMA 2017 mit Datenstand von 2016). Aus den Orthophotos wurden Waldflächen digitalisiert. Alle Flächen, die nach dieser Digitalisierungsprozedur noch keine Attribuierung erhielten, wurden als „Siedlungen und Sonstiges” erfasst. In einem letzten Arbeitsschritt wurden die Luftbilder aus Mitte des 20. Jahrhunderts rektifiziert und georeferenziert (Zeitschnitt 1960). Da diese ausschließlich schwarz-weiß vorlagen, wurden zuerst die leicht identifizierbaren Waldflächen digitalisiert. Acker- und Grünlandflächen waren hingegen schwer unterscheidbar. Deshalb wurden alle Flächen, die sowohl für 1820 als auch für 2015 als Acker ausgewiesen waren, ebenso für den Zeitschnitt 1960 als Acker angegeben. Mit Grünlandflächen wurde in derselben Weise verfahren.

Flächen, die nicht eindeutig zuordenbar waren, wurden in ihrem räumlichen Kontext einer „Best Guess”-Kategorie zugewiesen. Das bedeutet, dass Parzellen, in deren Nachbarschaft sich hauptsächlich Ackerland befand, ebenfalls als Ackerland klassifiziert wurden; analog wurde mit Grünland verfahren. Weitere Kriterien waren Seehöhe, Hangneigung und Distanz zu Siedlungen. Flache, niedrig gelegene Flächen in der Nähe von Siedlungen wurden als Acker gewertet; steile, höher gelegene und abgelegene Flächen als Grünland. Eine zusätzliche Bedingung war die Vorgabe, die Flächenstatistik der Gemeinde des betreffenden Jahres zu reproduzieren. Neben der Problematik der Schwarz-Weiß-Luftbilder bestanden weitere Herausforderungen: So sind insbesondere beim Zeitschnitt 1820 Wegverbindungen zwischen Siedlungen erkennbar, die teilweise heute im Wald liegen oder im aktuellen Wegenetz nicht mehr erkennbar bzw. zuordenbar sind. Räumliche Fehler durch Primärquellen und Digitalisierung schätzen wir auf 3-5 m für den Zeitschnitt 1820, 5-10 m für 1960 und weniger als 3 m für 2015.

Beim Prozess der Digitalisierung blieben lineare Strukturelemente und Kleinstrukturen, wie etwa Straßen- und Wegenetze, aber auch Hecken oder Baumreihen, systematisch unterrepräsentiert. Für die Landnutzungsklasse „Siedlungen und Sonstiges” bedeutet dies eine Unterschätzung von Siedlungen- und Verkehrsflächen außerhalb von Siedlungsgebieten, während innerhalb der Siedlungsgebiete die Siedlungsflächen aufgrund der Heterogenität überschätzt werden. Für unsere Untersuchungsgebiete nehmen wir an, dass sich die „sonstige Landnutzung” nicht signifikant veränderte (z. B. Gewässer, Tab. 2), sodass eine Änderung der Landnutzungsklasse „Siedlungen und Sonstiges” die Siedlungsflächenentwicklung anzeigt. 


\begin{tabular}{|c|c|c|}
\hline \multirow{2}{*}{$\begin{array}{l}\text { Landnutzungs- } \\
\text { klassen }\end{array}$} & Zeitschnitt 1820 & Zeitschnitt 2015 \\
\hline & $\begin{array}{c}\text { Kultur-Gattungen im } \\
\text { Franziszeischen Kataster* }\end{array}$ & InVeKoS, Orthophotos \\
\hline $\begin{array}{l}\text { Wald (Wald und } \\
\text { Waldweide) }\end{array}$ & $\begin{array}{l}\text { Laubholzwälder, Nadelholzwälder, Ge- } \\
\text { mischte Wälder, Kastanienwälder, Wiesen } \\
\text { mit Obstbäumen }\end{array}$ & Digitalisierung aus Orthophotos \\
\hline Grünland & $\begin{array}{l}\text { Trockene Wiesen, nasse Wiesen, } \\
\text { Weiden, Gemeindeweiden, Gestrüpp }\end{array}$ & $\begin{array}{l}\text { InVeKoS Feldstückkategorien: Grünland, } \\
\text { Almen, Weidefeldstücke }\end{array}$ \\
\hline Ackerland & $\begin{array}{l}\text { Obstgärten, Gemüsegärten, Weingärten, } \\
\text { Acker, Rott- oder Trisch-Äcker, Äcker mit } \\
\text { Obstbäumen, Äcker mit Weinreben, Äcker } \\
\text { mit Bäumen und Weinreben }\end{array}$ & InVeKoS Feldstückkategorie: Ackerland \\
\hline $\begin{array}{l}\text { Siedlungen und } \\
\text { Sonstiges }\end{array}$ & $\begin{array}{l}\text { Sümpfe, Sümpfe mit Rohrwuchs, Seen, } \\
\text { Teiche, Flüsse und Bäche, Öden und } \\
\text { nackte Felsen, Gebäude (alle Arten), Wege } \\
\text { (alle Arten) }\end{array}$ & $\begin{array}{l}\text { Restfläche bestehend u. a. aus: Versiegelte } \\
\text { Flächen (Gebäude, Verkehrsflächen, Park- } \\
\text { plätze, Gewerbegebiete, Betriebsflächen), } \\
\text { Gewässer, Abbauflächen (Steinbrüche, } \\
\text { Schottergruben), Gärten, Parks, Sportplät- } \\
\text { ze, naturnahe Flächen (Moore, Fels und } \\
\text { Geröll, Ruderalfluren) }\end{array}$ \\
\hline
\end{tabular}

* Nicht gelistete Kultur-Gattungen sind im Untersuchungsgebiet nicht vorhanden; Landnutzungsklassen des Zeitschnitts 1960 wurden über Digitalisierung der Luftbilder erfasst.

Tab. 2: Übersicht über die Einteilung und Erfassung der Landnutzungsklassen nach Zeitschnitt

Daten zu landwirtschaftlichen Erträgen (Getreide- und Wiesenertrag), zum Holzertrag und zur Bevölkerung wurden aus anderen Quellen zusammengestellt (Tab. 1) und in die Geodatenbank eingespeist. Erträge als „Outputs” aus dem land- und forstwirtschaftlichen Produktionssystem können Aufschluss über die Landnutzungsintensität geben (ERB et al. 2013) und werden zum Beispiel in der Agrargeschichte verwendet (vgl. LANGTHALER 2003).

Die Rekonstruktion der Landnutzungsgeschichte beider Fallstudien zeigte, dass in Waidhofen und Paldau zwar ähnliche Trends beobachtbar sind, diese aber eine sehr unterschiedliche Ausprägung aufweisen: Während die Fläche der Landnutzungsklasse „Siedlungen und Sonstiges“" in beiden Gemeinden deutlich zunahm (insbesondere in Paldau: $+16 \%$; in Waidhofen nur $+9 \%$ ), verringerten sich landwirtschaftliche Flächen (Paldau: $-14 \%$, Waidhofen: $-17 \%$ ). So wird in Paldau heute noch gemischte Landwirtschaft betrieben (37\% Ackerland und $10 \%$ Grünland), während in Waidhofen der Ackerbau nahezu komplett verschwunden ist (1\% Ackerland und $45 \%$ Grünland). Waldflächen haben in Waidhofen zugenommen (+8 \%) und in Paldau geringfügig abgenommen ( $-2 \%)$.

\section{Quantifizierung des Kulturlandschaftswandels}

Der flächenbezogene Kulturlandschaftswandel ist multidimensional. Für unsere Analyse entwickelten wir daher ein Indikatorensystem aus bereits erprobten Indikatoren, um die 
Entwicklung der Siedlungs-, Wald- und Landwirtschaftsflächen aufzuzeigen. Das Ziel bei der Indikatorenauswahl war es, eine möglichst umfassende Darstellung des Kulturlandschaftswandels unter Berücksichtigung der Datenverfügbarkeit für historische Zeiträume zu erreichen.

\subsection{Definition von Indikator und Indikatorensystem}

Flächenbezogene Fragestellungen sind häufig komplex und erfordern ein übergreifendes und zusammenhängendes Verständnis verschiedener Disziplinen (Wirtschaft, Politik, Sozial- oder Raumwissenschaften; HuANG et al. 2011). Über die Methodik der multikriteriellen Entscheidungsanalyse (MCDA, engl. multicriteria decision analysis) besteht die Möglichkeit, verschiedene Dimensionen und deren Bewertungskriterien mit einem gemeinsamen Ziel zu verknüpfen (MALCZEWSKI und RinNER 2015). Seit den 1980er Jahren hat hierbei die Hinzunahme räumlicher Daten oder geographischer Informationssysteme (GIS) zunehmend an Bedeutung gewonnen (HUANG et al. 2011; MALCZEWSKI und RINNER 2015). Im Kontext unserer Analyse greifen wir die Methodik einer MCDA in Form eines Indikatorensystems auf, indem wir die Bewertungskriterien als Indikatoren verstehen, mit dem Ziel, den Kulturlandschaftswandel zu quantifizieren.

Anlehnend an SiEdentop et al. 2007 definieren wir einen Indikator als wertfreie, deskriptive Mess- und Beobachtungsgröße mit dem inhärenten Anspruch der Komplexitätsreduktion. Erst im Kontext der Bezugnahme zu flächenpolitischen Zielen erhält der Indikator eine bewertende Aussage (SiEDEnTop et al. 2007). Werden mehrere Indikatoren verwendet und systematisch zusammengestellt, wird dieses Gebilde als Indikatorensystem verstanden.

\subsection{Indikatorenset des Kulturlandschaftswandels}

Es gibt zahlreiche Indikatoren und Indikatorensysteme, die eine Veränderung der Landschaft in ihrer Struktur oder Funktion unter ökologischen, ökonomischen oder sozialen Aspekten messen (z. B. in Deutschland Umwelt-Kernindikatoren des Umweltbundesamts (KIS), Indikatoren der Länderinitiative Kernindikatoren (LIKI) usw.; vgl. die Übersicht in Walz und Stein 2017, S. 60, oder GoetzKe 2018, S. 137). Wir fokussierten uns auf Indikatoren und Indikatorensysteme, die räumliche Trends der Kulturlandschaftsentwicklung auch hinsichtlich nachhaltiger Raumentwicklung widerspiegeln.

Die Indikatorenauswahl dieser Arbeit basierte auf den Untersuchungen von SIEDENTOP et al. (2007) und WALZ und STEIN (2017): SiEDENTOP et al. (2007) entwickelten den „Nachhaltigkeitsbarometer Fläche“ mit dem Ziel, qualitativ wie quantitativ die Flächennutzungsstruktur und deren Veränderungen auf verschiedenen räumlichen wie administrativen Ebenen zu bewerten. Darüberhinausgehend dient das Nachhaltigkeitsbarometer zur politischen Entscheidungsunterstützung und als eine Grundlage für die Steuerung der Flächennutzung. Es besteht aus 39 Indikatoren, die in vier Kategorien flächenpolitischer Ziele - die sogenannten Zielsysteme - eingeteilt werden: Reduktions- 
ziele, Erhaltungs- und Schutzziele, nutzungsstrukturelle Ziele und Nutzungseffizienzziele (Siedentop et al. 2007, S. 8-10, 40). Da der Fokus des Nachhaltigkeitsbarometers auf der Bewertung der Siedlungs- und Verkehrsflächenentwicklung liegt, wurde die Studie von Walz und SteIN (2017) hinzugezogen, um den Indikatoren-Pool des Barometers um Indikatoren für ein räumliches Monitoring des Landschaftswandels zu ergänzen (9 zusätzliche Indikatoren).

In Anlehnung an die von Walz und Stein (2017, S. 58) benannten, zum Kulturlandschaftswandel beitragenden Prozesse arbeiteten wir drei Entwicklungspfade heraus: 1. Siedlungsentwicklung, 2. Waldentwicklung und 3. Landwirtschaftsentwicklung. Diesen Pfaden wurden im folgenden Schritt aus dem vorhandenen Indikatoren-Pool geeignete Indikatoren zugeordnet (Tab. 3, Abb. 2). Die Auswahlkriterien waren hierbei die Datenverfügbarkeit in den gewählten Zeitschnitten, die Zuordenbarkeit des Indikandums (Ziel-, Problem- und Handlungsbezug) zu einem Entwicklungspfad und die Anwendbarkeit unabhängig von der Maßstabsebene, um einen Vergleich der Untersuchungsgebiete zu gewährleisten.

1. Siedlungsentwicklung: Die Siedlungsentwicklung zeigte die Veränderung der Siedlungs- und Verkehrsflächen im Untersuchungszeitraum an. Sie berechnete sich aus den Indikatoren der Flächeninanspruchnahme (S1), der Zersiedlung (S2), der Zerschneidung - berechnet als effektive Maschenweite (S3) und der Siedlungsdichte (S4). Die Zersiedlung ist ein schwer greifbares und bereits häufig definiertes Konstrukt, das mittlerweile als ein mehrdimensionales Phänomen verstanden wird. Nach JAEGER et al. (2018) umfasst die Definition im Wesentlichen die drei Bestandteile Siedlungsflächenausdehnung, Siedlungsflächenstreuung und Nutzungsdichte der Siedlungsflächen. In unserer Analyse griffen wir die ersten beiden Bestandteile des Zersiedlungsbegriffes auf und stellten sie über die Indikatoren Freiflächeneffizienz (S2.1) und Zerklüftungsgrad (S2.2) dar. Die geometrische Grundlage der Indikatorenberechnung war hierbei die Landnutzungsklasse „Siedlungen und Sonstiges“.

2. Waldentwicklung: Die Indikatoren der Waldentwicklung setzten sich aus der Waldfragmentierung - berechnet als effektive Maschenweite (W1) -, der Waldnutzungsintensität (W2) sowie dem Waldflächenanteil (W3) zusammen. Für die Berechnung der Indikatoren verwendeten wir die Landnutzungsklasse „Wald“.

3. Landwirtschaftsentwicklung: Für die Darstellung der Landwirtschaftsentwicklung verwendeten wir die Indikatoren Ökotone (L1), landwirtschaftliche Intensität (L2) sowie Grünlandanteil an der Landwirtschaftsfläche (L3). Die Berechnungsgeometrien bildeten die Landnutzungsklassen „Ackerland“ und „Grünland“. Für den Indikator Ökotone wurde die Randlänge zu den Geometrien der Waldflächen ermittelt.

Entsprechend der Indikatorenauswahl ließen sich nachfolgend die dazugehörigen heutigen flächenpolitischen Ziele oder Leitbilder einer nachhaltigen Raumentwicklung aus der Literatur zusammenfassen (BMLFUW 2002; GRUBER et al. 2018; ÖROK 2011; SIEDENTOP et al. 2007):

- Reduzierung der Flächenneuinanspruchnahme durch Siedlung und Verkehr (Z1).

- Räumliche Konzentration der Siedlungsentwicklung und Einbindung von Infrastruktursystemen in vorhandenen Siedlungsbestand (Z2).

- Nachverdichtung und kompakte Innenentwicklung (Z3). 
- Schutz und Erhalt von Boden (Z4), von unbebauten Bereichen, Freiräumen, Grünflächen und naturschutzfachlich bedeutsamen Flächen, der Artenvielfalt sowie des Erlebnis- und Erholungsraums für den Menschen (Z5).

- Erhaltung großflächiger unzerschnittener Landschaftsräume sowie Vermeidung von Zersiedlung (Z6).

- Umweltgerechte, extensive und den natürlichen Lebensraum schützende Land- und Forstwirtschaft (Z7).

Die Ziele können zueinander in Wechselwirkung stehen oder sich auch aus Konkretisierungen anderer Ziele ergeben (SIEDENTOP et al. 2007). Indikatoren unterschiedlicher Entwicklungspfade können daher durchaus gleichen Zielen zugeordnet werden (Tab. 3).

\begin{tabular}{|c|c|c|c|c|c|}
\hline Nr. & Bezeichnung & Beschreibung & Ziel** & $\mathrm{Gw}^{* * *}$ & Literaturverweis \\
\hline \multicolumn{6}{|c|}{ Siedlungsentwicklung (S) } \\
\hline $\mathrm{S} 1 *$ & $\begin{array}{l}\text { Flächeninan- } \\
\text { spruchnahme }\end{array}$ & $\begin{array}{l}\text { Anteil der Siedlungsfläche an der } \\
\text { Gesamtfläche (in \%) }\end{array}$ & $\begin{array}{l}\mathrm{Z} 1 \\
\mathrm{Z} 4\end{array}$ & 0,08 & $\begin{array}{l}\text { SIEDENTOP et al. } \\
2007, \text { S. } 60\end{array}$ \\
\hline $\mathrm{S} 2$ & Zersiedlung & & & & \\
\hline$S 2.1 *$ & $\begin{array}{l}\text { Freiflächen- } \\
\text { effizienz }\end{array}$ & $\begin{array}{l}\text { Beeinträchtigung des Lebensraum- } \\
\text { und Erholungspotenzials für Men- } \\
\text { schen und Tiere der von Siedlungen } \\
\text { umgebenen Freiflächen (\%) }\end{array}$ & $\begin{array}{l}\mathrm{Z} 1 \\
\mathrm{Z} 6\end{array}$ & 0,04 & $\begin{array}{l}\text { ACKERMANN et al. } \\
2013\end{array}$ \\
\hline$S 2.2$ & Zerklüftungsgrad & $\begin{array}{l}\text { Durchschnittliches Verhältnis des } \\
\text { Umfangs einer Siedlungsfläche zum } \\
\text { Umfang eines Kreises mit gleichem } \\
\text { Flächeninhalt }\end{array}$ & $\begin{array}{l}\mathrm{Z} 1 \\
\mathrm{Z} 6\end{array}$ & 0,04 & $\begin{array}{l}\text { SiEDENTOP et al. } \\
2007, \text { S. } 94\end{array}$ \\
\hline $\mathrm{S} 3$ & Zerschneidung & $\begin{array}{l}\text { Effektive Maschenweite (ha) als } \\
\text { Maß für die Größe unzerschnittener } \\
\text { Flächen }\end{array}$ & $\begin{array}{l}\mathrm{Z} 2 \\
\mathrm{Z} 6\end{array}$ & 0,08 & Moser et al. 2007 \\
\hline $\mathrm{S} 4$ & Siedlungsdichte & Bevölkerung pro $\mathrm{km}^{2}$ Siedlungsfläche & $\mathrm{Z3}$ & 0,08 & $\begin{array}{l}\text { SiEDENTOP et al. } \\
2007, \text { S. } 109\end{array}$ \\
\hline \multicolumn{6}{|c|}{ Waldentwicklung (W) } \\
\hline W1 & $\begin{array}{l}\text { Waldfragmentie- } \\
\text { rung }\end{array}$ & $\begin{array}{l}\text { Waldfragmentierung als effektive } \\
\text { Maschenweite (ha) (siehe S3) }\end{array}$ & $\mathrm{Z5}$ & 0,11 & $\begin{array}{l}\text { Moser et al. 2007; } \\
\text { WALZ und STEIN } \\
\text { 2017, S. 64-65 }\end{array}$ \\
\hline $\mathrm{W} 2 *$ & $\begin{array}{l}\text { Waldnutzungs- } \\
\text { intensität }\end{array}$ & $\begin{array}{l}\text { Holzertrag pro Fläche und Jahr (kg/ } \\
\text { ha/a) }\end{array}$ & $\mathrm{Z7}$ & 0,11 & \\
\hline W3 & Waldflächenanteil & $\begin{array}{l}\text { Anteil der Waldfläche an der Gesamt- } \\
\text { fläche }(\%)\end{array}$ & $\mathrm{Z5}$ & 0,11 & $\begin{array}{l}\text { WALZ und STEIN } \\
2017, \text { S. } 64\end{array}$ \\
\hline \multicolumn{6}{|c|}{ Landwirtschaftsentwicklung (L) } \\
\hline L1 & Ökotone & $\begin{array}{l}\text { Verhältnis der gemeinsamen Wald- } \\
\text { grenze zum Umfang der Landwirt- } \\
\text { schaftsfläche }\end{array}$ & $\begin{array}{l}\mathrm{Z} 5 \\
\mathrm{Z7}\end{array}$ & 0,11 & $\begin{array}{l}\text { WALZ und STEIN } \\
2017, \text { S. } 68\end{array}$ \\
\hline
\end{tabular}




\begin{tabular}{|c|l|l|c|c|c|}
\hline Nr. & \multicolumn{1}{|c|}{ Bezeichnung } & \multicolumn{1}{c|}{ Beschreibung } & Ziel** & Gw*** & Literaturverweis \\
\hline L2 & $\begin{array}{l}\text { Landwirtschaft- } \\
\text { liche Intensität }\end{array}$ & $\begin{array}{l}\text { Landwirtschaftsertrag pro Fläche; Ge- } \\
\text { treideertrag für Ackerland und Wie- } \\
\text { senertrag für Grünland (kg FW/ha) }\end{array}$ & Z7 & 0,11 & Z4 \\
\hline L3 & $\begin{array}{l}\text { Grünlandanteil } \\
\text { an der Landwirt- } \\
\text { schaftsfläche }\end{array}$ & $\begin{array}{l}\text { Anteil der Grünlandflächen an den } \\
\text { Landwirtschaftsflächen (\%) }\end{array}$ & Z5 & 0,11 & $\begin{array}{l}\text { WALZ und STEIN } \\
\text { 2017, S. 66 }\end{array}$ \\
\hline
\end{tabular}

*Aufsteigende Werte sind negativ zu bewerten; - ** Für die Definition der Ziele (Z) siehe die Erläuterungen im Fließtext auf Seite 265 unten bis Seite 266 oben; - *** Gesamtgewichtung (Gw) gemäß Gleichung (2), siehe Kapitel 3.3, gerundete Zahlen

Tab. 3: Übersicht über die ausgewählten Indikatoren

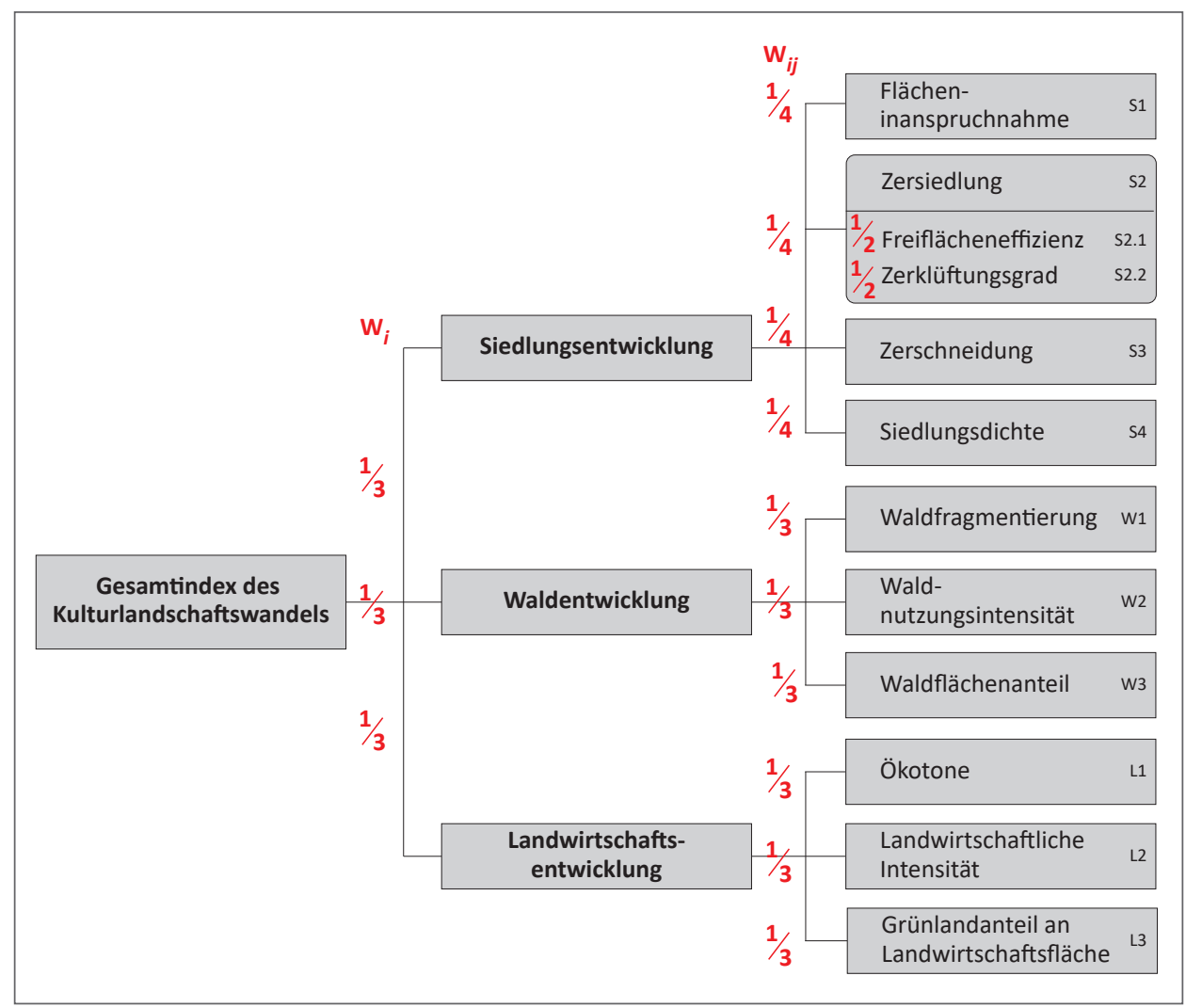

Rot: Gewichtung der Entwicklungspfade $\left(w_{i}\right)$ und Indikatoren $\left(w_{i j}\right)$ (siehe Gleichung 2$)$; zur Nummerierung der Indikatoren siehe auch Tabelle 3

Quelle: Eigene Darstellung

Abb. 2: Indikatorensystem zum nachhaltigen Kulturlandschaftswandel 


\subsection{Bilanzierung des Indikatorensystems}

Eine Grundvoraussetzung für die Bilanzierung eines Indikatorensystems ist die Übersetzung der verschiedenen Bewertungskriterien in eine vergleichbare Einheit, um diese über geeignete mathematische Verfahren anschließend verrechnen zu können (MALCZEWSKI und RinNer 2015). Die Bilanzierung des Indikatorensystems zur Quantifizierung des Kulturlandschaftswandels erfolgte nach dem Aggregierungsverfahren von SiEDENTOP et al. (2007, S. 121-130): Zunächst wurde jeder Indikator in einen Indexwert überführt. Hierbei wurde als Referenzwert $r$ für einen intrakommunalen Vergleich die Indizierung anhand eines Zeitschnittes (hier 1820), und für einen interkommunalen Vergleich der Gemeinden die Indizierung anhand des Durchschnittswertes aller Beobachtungsobjekte festgelegt.

Der Index $X$ eines Indikators $I$ kann mit der folgenden allgemeinen Berechnungsvorschrift normiert werden:

$$
\begin{gathered}
X=\left\{\begin{array}{c}
\max , \text { wenn } f(I, t, r, \max ) \geq \max \\
f(I, t, r, \max ) \\
\min , \text { wenn } f(I, t, r, \max ) \leq \text { min }
\end{array}\right. \\
\text { wobei }
\end{gathered}
$$

mit $t$ als Indikatortyp, der anzeigt, ob aufsteigende Indikatorwerte positiv oder negativ zu bewerten sind. Und mit $\min =0$ und $\max =200$ als untere beziehungsweise obere Indexgrenze, um Ausreißer zu vermeiden.

Indexwerte $<100$ zeigen demnach eine negativ gewertete Abweichung vom Referenzwert und Indexwerte $>100$ eine positiv bewertete Merkmalsausprägung an (SIEDENTOP et al. 2007, S. 123). Bei einem Vergleich zweier Indizes (z. B. eines Indikators verschiedener Zeitschnitte), ist der höhere Indexwert als positiver zu bewerten (d. h., „,bessere“ Kulturlandschaftsentwicklung).

Um eine ausgewogene Berechnung der Entwicklungspfade und des sich daraus ergebenden Gesamtindex des Kulturlandschaftswandels (GKLW) zu gewährleisten, wurde jeder Entwicklungspfad gleich gewichtet. Auch kam innerhalb eines Entwicklungspfades den Indikatoren die gleiche Bedeutung zu (vgl. Abb. 2). Die Bilanzierung ergab sich demnach wie folgt:

$$
G K L W=\sum_{i=1}^{n} w_{i} E_{i}=\sum_{i=1}^{n} w_{i} \sum_{j=1}^{m_{j}} w_{i j} X_{i j}
$$

wobei $n$ die Anzahl der Entwicklungspfade, $w_{i}$ die Gewichtung eines Entwicklungspfads $i$, $m_{j}$ die jeweilige Anzahl der Indikatoren $j, X_{i j}$ der normierte Indikator aus (1a) und $w_{i j}$ die Gewichtung von $X_{i j}$ ist. Die Gesamtgewichtung eines Indikators berechnete sich somit aus $w_{i} w_{i j}$ (Tab. 3, vgl. auch Abb. 2). 
Die Berechnung und Bilanzierung der Indikatoren wurde vollständig in der freien Entwicklungsumgebung und Programmiersprache R der Version 3.5.2 durchgeführt (R Core Team 2018). Gemäß der „Sichtweise der Historischen Geographie, Objekte der realen Welt als diskrete punktförmige, linien- oder flächenhafte Kulturlandschaftselemente anzusprechen“ (PLÖGER 1999, S. 106) und um Informationsverlust durch eine Datenkonvertierung in das Rasterformat zu vermeiden, berechneten wir die Indikatoren Vektoren-basiert. Die verwendeten Berechnungsvorschriften können in $\mathrm{R}$ über das frei-verfügbare Paket VLSM (Vector-based Landscape Metrics, siehe KNEVELS 2019) geladen werden.

\section{Ergebnisse}

Die Ergebnisdarstellung orientiert sich an den einzelnen Entwicklungspfaden und unterteilt sich in die intra- und interkommunale Bilanzierung, auch unter Bezugnahme auf die normierten Einzelindikatoren (Ergebnisübersicht nach Gesamtindex und Entwicklungspfad in Tab. 4).

\begin{tabular}{|c|c|c|c|c|}
\hline $\begin{array}{l}\text { Gemeinde und } \\
\text { Zeitschnitt }\end{array}$ & $\begin{array}{l}\text { Gesamt- } \\
\text { index }\end{array}$ & $\begin{array}{l}\text { Siedlungs- } \\
\text { entwicklung }\end{array}$ & $\begin{array}{c}\text { Wald- } \\
\text { entwicklung }\end{array}$ & $\begin{array}{c}\text { Landwirtschafts- } \\
\text { entwicklung }\end{array}$ \\
\hline \multicolumn{5}{|c|}{ Intrakommunale Bilanzierung } \\
\hline \multicolumn{5}{|c|}{ A: Paldau mit Referenzzeitschnitt Paldau 1820} \\
\hline Paldau 1820 & 100,00 & 33,33 & 33,33 & 33,33 \\
\hline Paldau 1960 & 68,56 & 18,24 & 29,41 & 20,91 \\
\hline Paldau 2015 & 32,41 & 2,60 & 21,12 & 8,70 \\
\hline \multicolumn{5}{|c|}{ B: Waidhofen mit Referenzzeitschnitt Waidhofen 1820} \\
\hline Waidhofen 1820 & 100,00 & 33,33 & 33,33 & 33,33 \\
\hline Waidhofen 1960 & 112,99 & 42,03 & 38,78 & 32,17 \\
\hline Waidhofen 2015 & 64,20 & 7,37 & 35,85 & 20,97 \\
\hline \multicolumn{5}{|c|}{ Interkommunale Bilanzierung } \\
\hline \multicolumn{5}{|c|}{ C: Vergleich der Gemeinden mit Durchschnittswert-Referenzierung (absteigend nach Gesamtindex) } \\
\hline Waidhofen 1960 & 149,27 & 58,16 & 42,73 & 48,38 \\
\hline Waidhofen 1820 & 130,49 & 47,14 & 37,16 & 46,19 \\
\hline Paldau 1820 & 117,01 & 46,20 & 26,78 & 44,04 \\
\hline Paldau 1960 & 86,62 & 34,90 & 25,52 & 26,20 \\
\hline Waidhofen 2015 & 83,31 & 16,04 & 40,50 & 26,77 \\
\hline Paldau 2015 & 26,06 & 3,36 & 14,27 & 8,43 \\
\hline
\end{tabular}

Tab. 4: Indikator-Bilanzierungsergebnisse: Gesamtindex und Differenzierung nach Entwicklungspfad 
Die folgende Übersicht zu den Indikatorwerten eines Zeitschnitts (Tab. 5) dient einem vertiefenden Verständnis der hier verglichenen Kulturlandschaftsentwicklung und eröffnet die Option einer In-Bezug-Setzung mit vergleichbaren Studien (vgl. Kapitel 5.3 und 5.4).

\begin{tabular}{|c|c|c|c|c|c|c|}
\hline \multirow{2}{*}{ Indikator } & \multicolumn{3}{|c|}{ Paldau } & \multicolumn{3}{|c|}{ Waidhofen } \\
\hline & 1820 & 1960 & 2015 & 1820 & 1960 & 2015 \\
\hline \multicolumn{7}{|c|}{ Siedlungsentwicklung (S) } \\
\hline $\mathrm{S} 1 *$ & 2,66 & 5,18 & 19,13 & 1,87 & 2,21 & 10,9 \\
\hline $\mathrm{S} 2.1$ & 59,14 & 48,67 & 17,60 & 61,55 & 71,86 & 31,31 \\
\hline $\mathrm{S} 2.2 *$ & 2,05 & 2,92 & 7,95 & 2,04 & 2,45 & 4,54 \\
\hline $\mathrm{S} 3$ & $3.626,91$ & $3.432,89$ & 87,35 & $3.220,94$ & $6.369,07$ & $1.234,53$ \\
\hline $\mathrm{S} 4$ & $2.997,85$ & $1.472,49$ & 416,56 & $3.235,42$ & $4.094,13$ & 800,18 \\
\hline \multicolumn{7}{|l|}{ Waldentwicklung (W) } \\
\hline $\mathrm{W} 1$ & 13,8 & 10,04 & 13,53 & 148,51 & 201,3 & 434,72 \\
\hline $\mathrm{W} 2 *$ & $2.237,95$ & $2.197,00$ & $5.666,67$ & $2.328,05$ & $2.197,00$ & $5.067,00$ \\
\hline W3 & 36,17 & 32,57 & 33,29 & 35,37 & 38,16 & 43,38 \\
\hline \multicolumn{7}{|c|}{ Landwirtschaftsentwicklung (L) } \\
\hline $\mathrm{L} 1$ & 0,59 & 0,23 & 0,04 & 0,46 & 0,63 & 0,09 \\
\hline $\mathrm{L} 2 *$ & $1.137,31$ & $4.021,54$ & $6.360,57$ & 755,19 & $3.808,9$ & $5.768,89$ \\
\hline L3 & 30,17 & 45,29 & 21,39 & 58,04 & 88,24 & 97,79 \\
\hline
\end{tabular}

* Aufsteigende Werte sind negativ zu bewerten.

Indikatoren: S1: Flächeninanspruchnahme; - S2.1: Freiflächeneffizienz; - S2.2: Zerklüftungsgrad; - S3: Zerschneidung; - S4: Siedlungsdichte; - W1: Waldfragmentierung; - W2: Waldnutzungsintensität; - W3: Waldflächenanteil; - L1: Ökotone; - L2: Landwirtschaftliche Intensität; - L3: Grünlandanteil an der Landwirtschaftsfläche

Tab. 5: Ergebnisse der ausgewählten Indikatoren

\subsection{Intrakommunale Bilanzierung}

Die Indikatorenbilanzierung für Paldau mit der Landnutzung von 1820 als Referenzzeitschnitt zeigte in allen Entwicklungspfaden und somit auch im Gesamtindex einen abfallenden Trend (Tab. 4, Teil A). So fiel der Gesamtindex im Untersuchungszeitraum um 67,59 Indexpunkte (IP) auf 32,41 IP.

Bei Betrachtung der Entwicklungspfade fällt auf, dass der Index der Siedlungsentwicklung mit -30,73 Indexpunkten die höchste Abnahme verzeichnete (Indexwert 2015: 2,66). Dies ergibt sich aus einer Flächenneuinanspruchnahme (S1) von etwa 16 Prozentpunkten und einer zunehmend zersiedelten sowie zerschnittenen Landschaft (Verdreifachung des Zerklüftungsgrades und Abnahme der effektiven Maschenweite um 98 Prozentpunkte; Abb. 3, vgl. Tab. 5 sowie Tab. A1 im Anhang). 
Die Landwirtschaftsentwicklung zeigte einen ähnlichen, aber weniger ausgeprägten Trend wie die Siedlungsentwicklung (-24,63 Indexpunkte auf 8,7 IP im Jahr 2015). Ausschlaggebend waren der hohe Rückgang der Ökotone von 0,59 auf 0,04 (-92,64 Indexpunkte) und die hohe Zunahme der landwirtschaftlichen Intensität (-100 Indexpunkte; seit 1820 mehr als verfünffacht) im Untersuchungszeitraum.
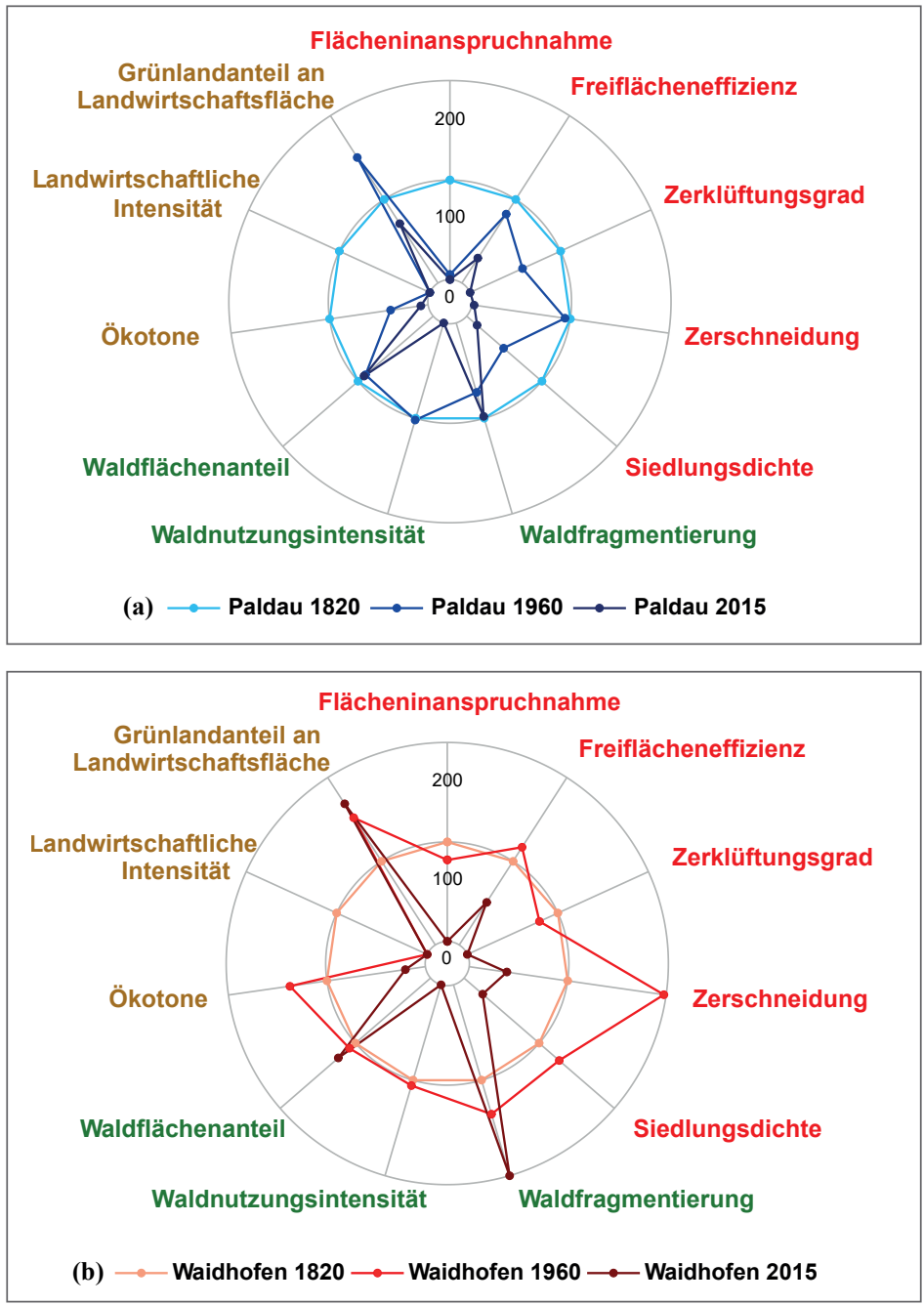

a: Paldau, Indizierung mit Paldau 1820; b: Waidhofen, Indizierung mit Waidhofen 1820; - Farbe der Entwicklungspfade: Waldentwicklung: grün, Siedlungsentwicklung: rot, Landwirtschaftsentwicklung: braun

Quelle: Eigene Darstellung

Abb. 3: Indizierung nach Referenzzeitschnitt 
Die Waldentwicklung wies mit -12,21 Indexpunkten den geringsten abfallenden Index zwischen dem 19. und 21. Jahrhundert auf. Während die Waldfragmentierung mit rund 14 ha und der Waldflächenanteil von etwa 33 Prozent über die Zeit nahezu konstant blieben, nahm die Nutzungsintensität des Waldes um mehr als das zweifache auf $5.667 \mathrm{~kg} / \mathrm{ha} / \mathrm{a}$ zu (vgl. Tab. 5).

Die Indikatorenbilanzierung für Waidhofen mit der Landnutzung von 1820 als Referenzzeitschnitt zeigte differierende Trends in den Entwicklungspfaden und im Gesamtindex (Tab. 4, Teil B). So war der Gesamtindex 1960 höher (Indexwert 112,99) und 2015 geringer (Indexwert 64,2) als zum Referenzzeitschnitt.

Einen ähnlichen Verlauf zeigte auch die Siedlungsentwicklung: Vom 19. Jahrhundert ausgehend stieg zunächst der Index um 8,7 Indexpunkte auf 42,03 IP an und fiel anschließend um 34,66 Indexpunkte auf den Indexwert von 7,37. Die hohe Abnahme von dem 20. zum 21. Jahrhundert erklärt sich durch eine zunehmende Zersiedlung (-72,78 Indexpunkte; -41 Prozentpunkte bei der Freiflächeneffizienz und Verdoppelung des Zerklüftungsgrades), erhöhte Flächeninanspruchnahme von mehr als 8 Prozentpunkten (-81,87 Indexpunkte) und eine abnehmende Siedlungsdichte um etwa 3.294 Einwohner pro $\mathrm{km}^{2}$ Siedlungsfläche (-101,81 Indexpunkte) (Abb. 3, vgl. Tab. 5 sowie Tab. A2 im Anhang).

Der Index der Waldentwicklung lag 1960 (Indexwert 38,78) und 2015 (Indexwert 35,85) höher als zum Referenzzeitschnitt (Indexwert 33,33). Zwar war die Waldnutzungsintensität im letzten Zeitschnitt etwa um das Zweifache höher als zu den vorherigen Zeitschnitten, jedoch hat der Waldflächenanteil um 5 bis 8 Prozentpunkte auf 43 Prozent zugenommen und die Waldflächen sind weiter zusammengewachsen (geringe Waldfragmentierung bzw. höhere effektive Maschenweite von 236 bis 289 ha; vgl. Tab. 5).

Die Landwirtschaftsentwicklung zeigte einen durchgehend abfallenden Trend: Der Indexwert ist von 33,33 Indexpunkten im Jahr 1820 um 12,36 IP auf 20,97 IP im Jahr 2015 gefallen. Auch wenn der Grünlandanteil mit 97,79 Prozent an der Landwirtschaftsfläche zum Zeitschnitt 2015 den höchsten Indexwert aufwies (168,49 Indexpunkte), waren der Rückgang der Ökotone von 0,46 bzw. 0,63 auf 0,09 (-79,72 Indexpunkte) sowie die erhöhte landwirtschaftliche Intensität (-100 Indexpunkte; seit 1820 mehr als versiebenfacht) entscheidend für den abfallenden Trend der Landwirtschaftsentwicklung.

\subsection{Interkommunale Bilanzierung}

Bei der interkommunalen Bilanzierung (Indizierung über den Durchschnitt als Referenzwert) zeigte sich, dass die Landnutzung Waidhofens 1960 mit 149,27 IP den höchsten Gesamtindex hatte, während die Landnutzung Paldaus 2015 den niedrigsten Wert aufwies (26,06 Indexpunkte; Tab. 4, Teil C). Überraschend ist, dass der Gesamtindex von Waidhofen 2015 lediglich um 3,31 Indexpunkte geringer ausfiel als von Paldau 1960, trotz der zeitlichen Differenz von 55 Jahren zwischen den Zeitschnitten. Auch wenn die Siedlungsentwicklung in Waidhofen zu diesem Zeitschnitt einen deutlich geringeren In- 
dexwert hatte (-18,86 Indexpunkte), so wurde dieses Defizit durch einen höheren Index in der Waldentwicklung (+14,98 Indexpunkte) ,,kompensiert“.

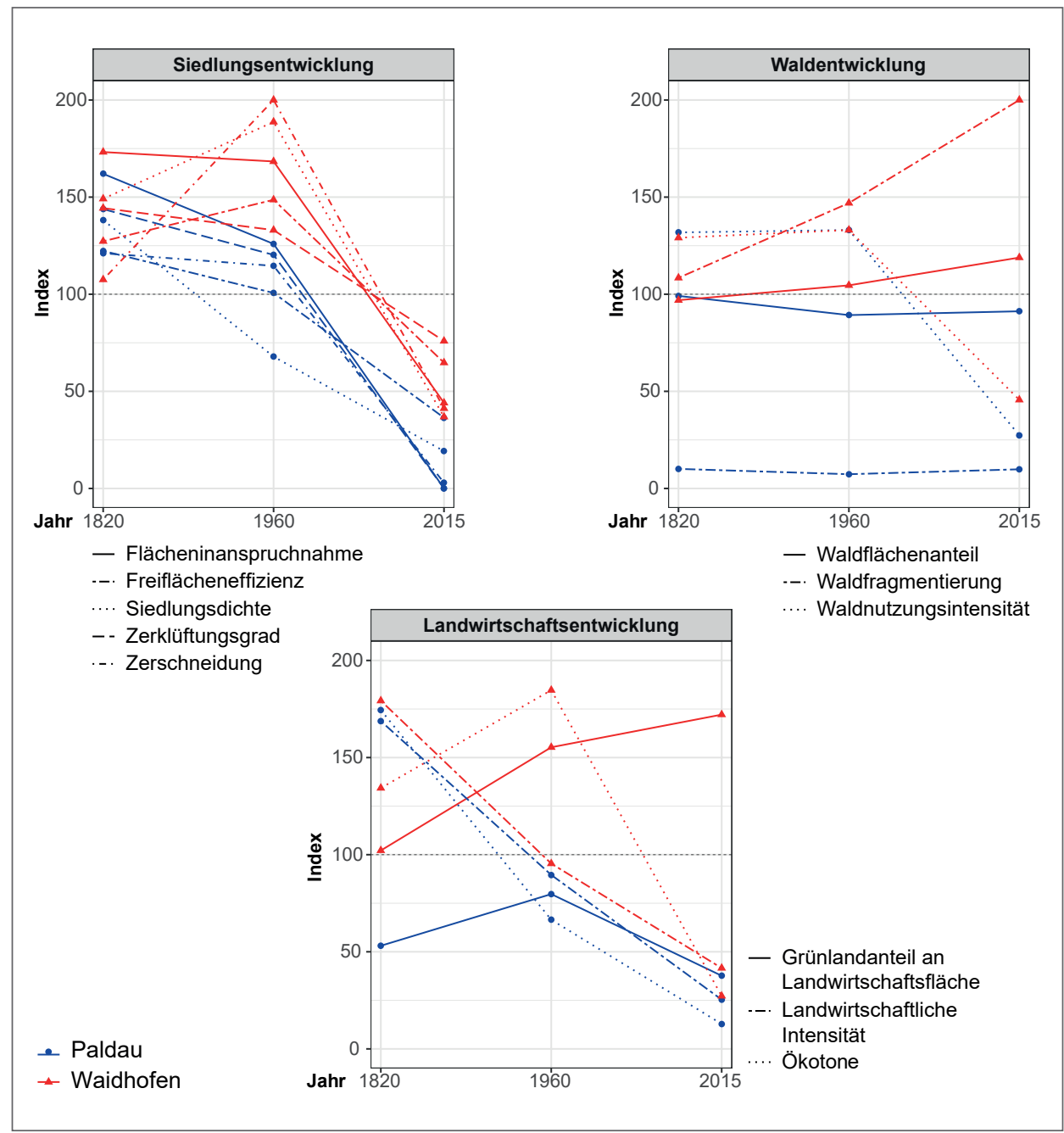

Quelle: Eigene Darstellung

Abb. 4: Vergleich beider Gemeinden über Durchschnittswert-Referenzierung. Farbe der Gemeinden: Paldau blau; Waidhofen rot.

Bei Betrachtung der einzelnen Indikatoren fällt auf, dass 70 Prozent der Indizes in Waidhofen über dem Referenzwert lagen (IP > 100), während dies in Paldau nur für etwa 39,4 Prozent zutraf (vgl. Abb. 4 sowie Tab. A3 im Anhang). Auch wies die Bilanzierung des Landnutzungsmusters Paldaus zum Zeitschnitt 2015 nahezu durchgehend den niedrigsten 
Indexwert auf. Der Kulturlandschaftswandel in Paldau ist somit im Vergleich mit Waidhofen negativer zu bewerten.

\section{Diskussion}

\subsection{Kulturlandschaft im Wandel}

Wir konnten zeigen, dass Indikatoren, die zum Monitoring rezenter Landschaftsprozesse konzipiert wurden, auch wenn sie auf historische Landnutzungsdaten angewendet werden, aussagekräftige Ergebnisse bzw. Aussagen über Trends erzielen. In beiden Fallstudien hat der Gesamtindex bei der intrakommunalen Bilanzierung seit 1820 abgenommen (Paldau: -67,59 Indexpunkte, Waidhofen: -35,8 Indexpunkte), was eine negativ gewertete Kulturlandschaftsentwicklung darstellt.

Die Waldentwicklung zeigte in den Gemeinden unterschiedliche Trends (Paldau: -12,21 Indexpunkte, Waidhofen: +2,52 Indexpunkte). Wir vermuten, dass sich die Veränderung des Waldflächenanteils (W3) und das Zusammenwachsen des Waldes (W1) durch die Konzentration der Landwirtschaft auf Gunstlagen und folglich reduzierten Druck auf Waldflächen erklären lässt (vgl. Phase der „Übergangsgesellschaft“ in BÄTZING 2002; 2017). Als Effekt blieben die Indikatorwerte für W1 und W3 für Paldau nahezu konstant, während für Waidhofen eine Zunahme zu verzeichnen war. Wälder bieten bedeutenden Lebensraum für Tiere und Pflanzen sowie Erholungsraum für den Menschen, sie bieten in Gebirgslagen Schutz vor Naturgefahren und ihr Wachstum trägt zum Klimaschutz bei (GöTZL et al. 2015). Auf den Index der Waldentwicklung wirkte jedoch seit den 1960er Jahren die zunehmende Nutzungsintensität des Waldes (W2) der positiven Entwicklung von W1 und W3 entgegen.

Die landwirtschaftlichen Flächen stellen eine prägende Landnutzungsklasse in beiden Untersuchungsgebieten dar und waren im Untersuchungszeitraum durch Mechanisierung, Rationalisierung, Intensivierung und biologisch-technische Fortschritte (Düngung, Pflanzenzucht, Pflanzenschutz, etc.) einem erheblichen Strukturwandel unterworfen (vgl. HAMPICKE 2013, S. 18-21). In beiden Untersuchungsgebieten verzeichnete die Landwirtschaftsentwicklung eine durchgehend abnehmende Tendenz (Paldau: -24,63 Indexpunkte, Waidhofen: -12,36 Indexpunkte). Insbesondere die Abnahme seit 1960 zeigt die Fortsetzung der Segregation in „Produktionslandschaften“ und „Stilllegungslandschaften“ (vgl. LinSER et al. 2005) mit einer zunehmenden Intensivierung von Gunstflächen (L2, vgl. Phase der „Dienstleistungsgesellschaft“ in BäTZING 2002; 2017).

Die Siedlungsentwicklung wies in beiden Untersuchungsgebieten einen ähnlich hohen Negativtrend auf (Paldau: -30,73 Indexpunkte, Waidhofen: -25,96 Indexpunkte). Insbesondere seit 1960 nahmen die Flächenneuinanspruchnahme (S1), die Zersiedelung (S2) und Zerschneidung (S3) der Landschaft deutlich zu - charakteristisch für die von BÄTZING (2002; 2017) beschriebene Phase der „Dienstleistungsgesellschaft“. Die Gemeinde Waidhofen war im Untersuchungszeitraum geringer ,urbanisiert“ als Paldau, was die alpine Ausprägung dieses Gebietes unterstreicht. Dies verdeutlicht auch die Gegenüberstellung beider Gemeinden durch die interkommunale Bilanzierung, in der Waidhofen tendenziell 
höhere Indexwerte aufwies (Indizes über dem Durchschnittswert: Waidhofen: 70 \%, Paldau: $37,7 \%$ ).

Bei Betrachtung und Einordnung ausgewählter Indikatorwerte und ihrer langfristigen Trends zeigen sich Übereinstimmungen mit vergleichbaren Studien trotz abweichender Ausgangsdaten und Zeiträume: So ist die Abnahme der effektiven Maschenweite (siehe S3, Zerschneidung) in Waidhofen (ca. $62 \%$ ) vergleichbar mit den von BERTILLER et al. (2007) veröffentlichten Werten für die Schweiz (56 bis $70 \%$ ); während die Abnahme Paldaus (ca. 98 \%) deutlich stärker ausfällt (vgl. Tab. 5). Die Tendenz der Vervielfachung der Flächeninanspruchnahme (S1) der Untersuchungsgebiete (Waidhofen: Versechsfachung, Paldau: Versiebenfachung; vgl. Tab. 5) zeigte sich auch in anderen Studien (z. B. Vervierfachung bei TASSER et al. 2009, S. 620, oder Versechsfachung in BENDER et al. 2005, S. 120). Auch lässt sich die Veränderung des Waldflächenanteils (W3) in seiner Tendenz (Waidhofen: +8 Prozentpunkte, Paldau: -2.88 Prozentpunkte) in der geeigneten Ökoregion von TASSER et al. (2009, S. 621) wiederfinden (landwirtschaftlich genutzte Hänge der montanen Höhenstufe: geschätzt +12 Prozentpunkte; landwirtschaftlich genutzte Täler der kollinen Höhenstufe: geschätzt -1 Prozentpunkt).

\subsection{Daten und Indikatorenauswahl in der Retrospektive}

Wir verwendeten verschiedene Datenquellen unterschiedlicher Qualität in Bezug auf die räumliche, zeitliche und thematische Auflösung (vgl. Tab 1). Für den Zeitschnitt 1820 lagen uns verlässliche Bevölkerungsdaten auf Gemeindeebene erst mit dem Beginn der ,statistischen Phase" der Siedlungsforschung ab der Volkszählung 1869 vor (vgl. KLEIN 1990, S. 57; Statistik Austria 2011a; 2011b). Für den Zeitraum von 1821 bis 1857 schätzt KLEIN (1973), dass die Bevölkerung in der Steiermark um 170.000 Einwohner auf 670.000 und in Niederösterreich (mit Wien) um 530.000 Einwohner auf eine Bevölkerungszahl von 1,67 Millionen angestiegen ist (Tabelle 1 in KLEIN 1973, S. 105). Wir nehmen daher an, dass wir die Bevölkerungszahl für den Zeitschnitt 1820 leicht überschätzen.

Für den Zeitschnitt 1960 lagen die verfügbaren Schwarz-Weiß-Luftbilder für die Gemeinden um neun Jahre auseinander (Paldau 1953, Waidhofen 1962), sodass Landschaftsveränderungen im Zuge des „Wirtschaftswunders“ ab 1955 (vgl. BäTZING 2017, S. 401) in Paldau unterrepräsentiert sein könnten. Auch war die Interpretation der Schwarz-Weiß-Luftbilder besonders herausfordernd: So ließen sich aufgrund der geringen Bildqualität die Landnutzungsklassen Grünland und Ackerland nur mit einer hohen Unsicherheit unterscheiden. Zusätzliche Quellen zu diesem Zeitschnitt zur Unterstützung der Digitalisierung wie zum Beispiel die Österreichische Karte (ÖK) wären wünschenswert gewesen (vgl. BENDER 2007). Die räumliche Datenqualität für die Jahre 1820 bzw. 2015 war hingegen aufgrund der parzellen- bzw. katastergenauen Angaben für unsere Analyse sehr gut geeignet. Des Weiteren war insbesondere die thematische Auflösung der Datenquellen zu den forst- und landwirtschaftlichen Erträgen sehr heterogen. So lagen Informationen teilweise nur auf der räumlich-statistischen Bezugseinheit der Bundesländer (z. B. Forstinventur für Zeitschnitt 2015) oder Nationalstaaten (z. B. WeIss et al. 2000 für den Zeitschnitt 1960) vor. Indikatorwerte von Zeitschnitten mit solch aggregierten 
Daten sind für den interkommunalen Vergleich zwar nur begrenzt aussagekräftig (z. B. für W2, Waldnutzungsintensität), erlauben jedoch, Aussagen über langfristige Trends zu treffen.

Digitalisierungsfehler bei der Überführung der historischen Quellen in ein GIS konnten durch die Bearbeitung durch nur eine einzige zuständige Person minimiert werden. Diese Zuständigkeit gewährleistete zudem durch die gewonnene Expertise zunehmende Vertrautheit mit den örtlichen Gegebenheiten und verringerte Interpretationsfehler. Die fehlende Berücksichtigung von linearen Strukturelementen (z. B. Straßen und Wegenetze) kann zu einer Unterschätzung der Siedlungsflächen und somit zu einer Überschätzung der Siedlungsentwicklung und folglich des Gesamtindexes geführt haben. Wir vermuten, dass die genannten Beeinträchtigungen (Unterrepräsentation linearer Strukturelemente, Schwarz-Weiß-Luftbilder 1960) den vergleichsweisen hohen Gesamtindex für Waidhofen 1960 (intrakommunal: 112,99 Indexpunkte, interkommunal: 149,27 Indexpunkte) mitverursacht haben könnten.

Die Auswahl der Indikatoren und deren ausgewogene Zusammenstellung zu einem Indikatorensystem ist nur eine Möglichkeit von vielen zur Quantifizierung des Kulturlandschaftswandels. Die Indikatorenzuordnung zu den Entwicklungspfaden ist per se ein wertender Vorgang, sodass je nach Auswahl und Zuweisung unterschiedliche Aspekte des Beschreibungsgegenstandes in den Vordergrund gestellt und in die Bilanzierung eingebracht werden können (SIEDENTOP et al. 2007, S. 49). Auch können beim Verfahren der Indizierung wissenschaftlich oder politisch begründete Transformationsfunktionen anstatt eines Durchschnittswertes oder eines Referenzzeitschnitts verwendet werden (SIEDENTOP et al. 2007, S. 121). Ebenso kann die additive Zusammenstellung, wenn sie durch eine andere Gewichtungsmatrix ersetzt wird, zu anderen Ergebnissen führen.

Weitere für den Kulturlandschaftswandel bedeutsame Indikatoren blieben aufgrund fehlender Datenbasis (z. B. fehlende Statistiken) oder der Möglichkeit von Fehlinterpretation (z. B. Shannon-Diversität) unberücksichtigt. So könnten Aspekte des sozialen und wirtschaftlichen Wandels in Folgeuntersuchungen hinzugezogen werden (z. B. Indikatoren zu Globalisierung, Wohlstandsentwicklung, Modernisierung, etc.; vgl. BENDER et al. 2002). Auch könnten die naturgegebenen, landschaftsprägenden topographischen Gegebenheiten - beispielsweise durch ein digitales Geländemodell dargestellt - bei einer bundesweiten Untersuchung weitere zu berücksichtigende Faktoren einbringen. HeILAND et al. (2003, S. 25) weisen jedoch darauf hin, dass eine vollständige, integrierende Zusammenschau ökonomischer, ökologischer und sozialer Aspekte keinem Indikatorensystem gelinge. Die Landesanstalt für Umwelt, Messungen und Naturschutz Baden-Württemberg führte hierzu aus, dass ,ein Gesamturteil [...] subjektiv in Form einer argumentierenden Zusammenfassung, nicht aber als objektiv erhobene oder errechnete Gesamtmesszahl möglich [ist]“ (LUBW 2007, S. 4).

Die Bewertung der Indikatoren ist zudem nicht immer eindeutig. So fließt der Indikator der Waldfragmentierung mit größeren, nicht-fragmentierten Waldflächen positiv in die Bilanzierung ein (höherer ökologischer Nutzen als kleine, mosaikartige Wälder). Dies wird jedoch im Diskurs der Verwaldung bzw. Verbuschung der alpinen Landschaft vor allem mit Bezug zum Tourismus nicht überall positiv bewertet („,Disneylandisierung“ oder „Musealisierung“ der Alpen; vgl. BORSDORF und BENDER 2007; GÜTHLER 2008). 


\subsection{Umsetzung und Übertragbarkeit}

Durch die Berechnung eines Gesamtindex werden Kulturlandschaftsveränderungen unterschiedlicher multikriterieller Entwicklungspfade quantifizierbar gemacht und somit in ihrem langfristigen Trend verdeutlicht. Allerdings bildet der Gesamtindex lediglich Kulturlandschaftsveränderungen aus Momentaufnahmen ab und übersieht daher Landnutzungswechsel im zeitlichen Kontinuum zwischen den Zeitschnitten. Auch sind die Unsicherheiten in der abweichenden Qualität der Datenquellen zwischen Zeitschnitten und im Indikatorenverständnis (vgl. Kapitel 5.2) per se in der Aussagekraft des Gesamtindex enthalten. Unsicherheiten in historischen Datenquellen sind teilweise unbekannt oder nur schwierig zu beziffern (Plewe 2002). Um Unsicherheiten in der Aussagekraft des Gesamtindex zu reduzieren, könnten in Folgeuntersuchungen Trendeinschätzungen zu einem Referenzjahr formuliert werden (z. B. zunehmend, konstant, abnehmend), welche über einen ordinal-skalierten (Gesamt)Index (z. B. sehr schwach bis sehr stark) in ihrer Merkmalsausprägung beschrieben werden (vgl. SIEDENTOP et al. 2007, S. 122, 137).

Es gibt bisher keinen festgeschriebenen Standardsatz von Indikatoren zur Beschreibung und zum Monitoring des Kulturlandschaftswandels (WALZ 2013). Das vorgestellte Indikatorensystem wurde unter Berücksichtigung der Datenbasis und der getroffenen Annahmen erstellt. Aufgrund des Indizierungsverfahrens (Durchschnittswert und Referenzzeitschnitt) können die Indexwerte nicht ohne Weiteres mit Ergebnissen anderer Untersuchungen verglichen werden. Die nicht-indizierten Indikatorwerte (Tab. 5) können jedoch bei gegebener Skalenunabhängigkeit mit anderen Gebieten in Bezug gesetzt werden. Zudem ermöglicht der Fokus auf wenige Landnutzungsklassen eine einfache Fortschreibung der Analyse. In Anbetracht der Herausforderungen in der Datenbeschaffung und -erzeugung (vgl. Kapitel 5.2) ist eine landesweite Umsetzung eher als echtes Desiderat als eine realistische Überlegung zu betrachten.

Die vorgestellte Methode zur Quantifizierung des Kulturlandschaftswandels kann als Grundlage für Untersuchungen in anderen Gemeinden und Bundesländern dienen. Die Berechnungsvorschriften der Indikatoren stehen in der freien Programmierumgebung über das R-Paket VLSM (KNEvels 2019) zur Verfügung und können somit in vergleichbaren Untersuchungen verwendet werden.

\subsection{Anwendungsmöglichkeiten der historischen Analyse}

Durch die Anwendung von Indikatoren mit raumplanerischer Relevanz (z. B. S3, Landschaftszerschneidung) bietet sich die Möglichkeit, den historischen Indikatorwert als Ansatzpunkt für Zielvereinbarungen oder sachorientierte Entscheidungen auf Planungsebene heranzuziehen (z. B. Festsetzung von Grenzwerten, Zielvorgaben in Wenn-Dann-Szenarien, Bestimmung von zukünftiger Flächennutzung).

Eine weitere Anwendung der Ergebnisse ist die Verknüpfung mit Empfindlichkeitsuntersuchungen (BERTILLER et al. 2007). So könnte überprüft werden, ob der negative Trend in der Siedlungs- oder Landwirtschaftsentwicklung mit Bestandsrückgängen von Pflanzen- oder Tierarten korreliert (vgl. ROEDENBECK und KöHLER 2006). Auch könnten 
über expertenbasierte oder zufällige Flächenumwidmungen in Simulationen jene Flächen identifiziert werden, deren Entwicklung sich besonders negativ (oder positiv) auf einen Indikator oder eine Landschaftsfunktion (Ökosystemdienstleistung) auswirkt. Die Erkenntnisse könnten erneut in planerische und politische Entscheidungen fließen (z. B. Schwerpunktbereiche für Siedlungsrückbau oder Ausweisung von Schutzgebieten).

Eine Bewertung der raumplanerischen Wirksamkeit vergangener politischer Maßnahmen oder Strategien ist hinsichtlich des Zeithorizonts mit dem vorgestellten Ansatz nur bedingt möglich und hier auch nicht beabsichtigt: Naturschutzgesetze gibt es in Österreich erst seit dem 20. Jahrhundert (Waidhofen: Niederösterreichisches Naturschutzgesetz 1924; Paldau: Steirisches Naturschutzgesetz 1976). In unserer Analyse decken wir Zeiträume ab, die zeitlich vor der Definition oder Berücksichtigung von Kriterien nachhaltiger Raumentwicklung liegen. Somit bewerten wir nicht die Wirksamkeit politischer Maßnahmen oder Strategien, sondern zeigen den Effekt historischer Prozesse auf eine auf heutige Perspektive bezogene (nicht-) nachhaltige Raumentwicklung auf.

\section{Fazit und Ausblick}

Wir konnten mithilfe eines Indikatorensystems quantitativ belegen, wie sich die Kulturlandschaft in beiden Untersuchungsgebieten in ihrer Flächennutzung seit der Industrialisierung stark verändert hat. Der Gesamtindex des Kulturlandschaftswandels ist ausgehend von 1820 deutlich gesunken. Ausschlaggebend hierfür waren vor allem die Zunahme von Siedlungs- und Verkehrsflächen sowie die intensivere Nutzung von Wald- und Landwirtschaftsflächen.

Die Anwendung eines auf räumlichen Informationen basierenden Indikatorensystems liefert über die reine Flächenstatistik hinausgehend interessante Rückschlüsse auf die langfristige Entwicklung eines Gebiets. Zwar können räumlich explizite Daten nur einen Teil der historischen Flächenentwicklung von Kulturlandschaften nachzeichnen, es war jedoch möglich, BäTZINGs $(2002 ; 2017)$ qualitativ beschriebene Phasen des Kulturlandschaftswandels im Alpenraum nachzuvollziehen und für zwei Gemeinden zu quantifizieren. Dieser historische Blick ermöglicht eine Grundlage für sachorientierte Entscheidungen auf Planungsebene: So können Kontexte für rezente Planungsentscheidungen geschaffen werden (z. B.: „Wo sollten künftig welche Nutzungen erlaubt sein, um eine Landschaftszerschneidung von diesem Niveau oder jenem Jahr zu erreichen.“). Auch dienen die Daten zur Überprüfung der Plausibilität von Wenn-Dann-Szenarien (z. B.: „Wenn wir jenen Wert bei der Waldfragmentierung erreichen wollten, dann müssten pro Jahr x Hektar aufgeforstet werden."). Zudem vermittelt die Quantifizierung vergangener landschaftsprägender Prozesse relevante Erkenntnisse über historische Zusammenhänge sozio-geographischer Strukturen (z. B. Struktur des Siedlungswachstums, landwirtschaftliche Umstrukturierung).

Strukturelle Veränderungen der Landschaft verlaufen meist schleichend, sodass erst ein Monitoring eines längeren Zeitraums Ausmaße von Veränderungen erkennbar macht (WALz 2013). Ein erweiterter Indikatorensatz mit sozialen und ökologischen Aspekten (z. B. zu Konsum, Tourismus, Verkehr oder Abfall) und mit Einbezug der Topographie des Gebietes (z. B. zu Ästhetik, Berücksichtigung bautechnischer Restriktionen, Naturgefah- 
ren, Habitatkonfiguration) könnte in Zukunft helfen, die Darstellung einer nachhaltigen Raumentwicklung noch weiter zu fassen.

\section{Hinweis und Danksagung}

Die vorliegende Studie ist im Rahmen des ILLAS-Projekts („Integrating Land Use Legacies in Landslide Risk Assessment to Support Spatial Panning") entstanden und wurde durch das ACRP („Austrian Climate Research Program“) gefördert (ACRP9-ILLASKR16AC0K13226). Der Beitrag von S. GINGRICH wurde zudem durch den Europäischen Forschungsrat finanziell unterstützt (ERC-2017-StG 757995 HEFT). Wir möchten uns bei vier anonymen Gutachterinnen und Gutachtern sowie dem Schriftleiter für die konstruktiven und wertvollen Kommentare bedanken.

\section{Literaturverzeichnis}

Ackermann W., Schweiger M., Sukopp U., Fuchs D., Sachteleben J. (2013): Landschaftszersiedlung. In: Ackermann W., Schweiger M., Sukopp U., Fuchs D., Sachteleben J., Bundesamt für Naturschutz (Hrsg.): Indikatoren zur biologischen Vielfalt. Entwicklung und Bilanzierung. Münster: Landwirtschaftsverlag, S. 186-198 (= Naturschutz und Biologische Vielfalt, 132).

AMA - Agrarmarkt Austria (2017): Katalog INVEKOS Schläge Österreich. Datenstand der zeitlichen Ausdehnung von 2016. Wien: AMA. - https://www.data.gv.at/katalog/dataset/ e21a731f-9e08-4dd3-b9e5-cd460438a5d9 (letzter Zugriff: 20.05.2020).

BäTZING W. (2001): Zur Abgrenzung der Alpen durch die Alpenkonvention. In: Jahrbuch des Vereins zum Schutz der Bergwelt, 66, S. 29-34.

BäTZING W. (2002): Die aktuellen Veränderungen von Umwelt, Wirtschaft, Gesellschaft und Bevölkerung in den Alpen. Berlin: Umweltbundesamt. - https://www.umweltbundesamt.de/sites/ default/files/medien/publikation/long/2150.pdf(letzter Zugriff: 20.05.2020).

BäTzING W. (2017): Die Alpen - tiefgreifende Nutzungsveränderungen als Herausforderung für den Naturschutz. In: Natur und Landschaft, 92 (9), S. 398-406. - https://doi.org/10.17433/9 .2017.50153497.398-406.

Bender O. (2003): Struktur und Dynamik der Kulturlandschaft. Diskussion (neuer) Methoden und Anwendungen einer diachronischen Landschaftsanalyse. In: Mitteilungen der Österreichischen Geographischen Gesellschaft, 145, S. 119-146.

BeNDER O. (2007): Analyse der Kulturlandschaftsentwicklung der Nördlichen Fränkischen Alb anhand eines katasterbasierten Geoinformationssystems. Leipzig: Deutsche Akademie für Landeskunde (= Forschungen zur Deutschen Landeskunde, 255).

Bender O., Borsdorf A., Pindur P., Pöckl A., Vorauer K. (2002): Räumlicher Strukturwandel in den Alpen. Zur Problematik von alpinen Raumbeobachtungs- und -informationssystemen. In: Mitteilungen der Österreichischen Geographischen Gesellschaft, 144, S. 37-58.

Bender O., Boehmer H. J., Jens D., Schumacher K. P. (2005): Using GIS to Analyse Long-term Cultural Landscape Change in Southern Germany. In: Landscape and Urban Planning, 70 (1-2), S. 111-125. - https://doi.org/10.1016/j.landurbplan.2003.10.008.

Bertiller R., SCHWick C., JAeGer J. (2007): Landschaftszerschneidung Schweiz. Zerschneidungsanalyse 1885-2002 und Folgerungen für die Verkehrs- und Raumplanung. Bern: Bundesamt für Straßen (=ASTRA-Bericht, 1175). 
BGB1. - Bundesgesetzblatt (1992): Bundesgesetz, mit dem Maßnahmen zur Sicherung der Ernährung sowie zur Erhaltung einer flächendeckenden, leistungsfähigen, bäuerlichen Landwirtschaft getroffen werden (Landwirtschaftsgesetz 1992 - LWG). Wien: Verlag der Österreichischen Staatsdruckerei (= Bundesgesetzblatt, Jg. 1992, 128. Stück vom 30.06.1992, 375. Bundesgesetz). - https://www.ris.bka.gv.at/Dokumente/BgblPdf/1992_375_0/1992_375_0. pdf(letzter Zugriff: 20.05.2020).

BGBl. - Bundesgesetzblatt (1995): Übereinkommen zum Schutz der Alpen (Alpenkonvention). Wien: Verlag der Österreichischen Staatsdruckerei (= Bundesgesetzblatt, Jg. 1995, 151. Stück vom 21.07.1995, 477. Bundesgesetz). - https://www.ris.bka.gv.at/Dokumente/BgblPdf/1995 _477_0/1995_477_0.pdf(letzter Zugriff: 20.05.2020).

BMLFUW - Bundesministerium für Land- und Forstwirtschaft, Umwelt und Wasserwirtschaft (2002): Die österreichische Strategie zur Nachhaltigen Entwicklung. Österreichs Zukunft nachhaltig gestalten. Wien: BMLFUW (heute Bundesministerium für Nachhaltigkeit und Tourismus). - https://www.bmnt.gv.at/dam/jcr:546785f3-7333-488d-a2b2-4c0f50a49190/ NSTRAT\%202002.pdf (letzter Zugriff: 20.05.2020).

BORSDORF A., BENDER O. (2007): Kulturlandschaftsverlust durch Verbuschung und Verwaldung im subalpinen und hochmontanen Höhenstockwerk: Die Folgen des klimatischen und sozioökonomischen Wandels. In: Aistleitner J. (Hrsg.): Alpine Kulturlandschaft im Wandel. Hugo Penz zum 65. Geburtstag. Innsbruck: Geographie Innsbruck / Innsbrucker Geographische Gesellschaft, S. 29-50.

Erb K.-H., Haberl H., Jepsen M. R., Kuemmerle T., Lindner M., Müller D., Verburg P. H., ReEnberg A. (2013): A Conceptual Framework for Analysing and Measuring Land-use Intensity. In: Current Opinion in Environmental Sustainability, 5 (5), S. 464-470. - https://doi. org/10.1016/j.cosust.2013.07.010.

Esswein H., Jaeger J., Schwarz-von Raumer H.-G., Müller M. (2002): Landschaftszerschneidung in Baden-Württemberg. Zerschneidungsanalyse zur aktuellen Situation und zur Entwicklung der letzten 70 Jahre mit der effektiven Maschenweite. Stuttgart: Akademie für Technikfolgenabschätzung in Baden-Württemberg (= Arbeitsbericht, 214).

Gingrich S., Erb K.-H., Krausmann F., Gaube V., Haberl H. (2007): Long-term Dynamics of Terrestrial Carbon Stocks in Austria: A Comprehensive Assessment of the Time Period from 1830 to 2000. In: Regional Environmental Change, 7 (1), S. 37-47. - https://doi. org/10.1007/s10113-007-0024-6.

GoETZKe R. (2018): Indikatoren zur Bewertung einer nachhaltigen und klimawandelgerechten Siedlungsentwicklung. In: Behnisch M., Kretschmer O., Meinel G. (Hrsg.): Flächeninanspruchnahme in Deutschland. Berlin: Springer, S. 131-154. - https://doi.org/10.1007/9783-662-50305-8_8.

Götzl M., Schwaiger E., Schwarzl B., Sonderegger G. (2015): Ökosystemleistungen des Waldes. Erstellung eines Inventars für Österreich. Wien: Umweltbundesamt (= Report, 0544). http://www.umweltbundesamt.at/fileadmin/site/publikationen/REP0544.pdf (letzter Zugriff: 20.05.2020).

Gross M. (2003): Beitrag zur Lithostratigraphie des Oststeirischen Beckens (Neogen/Pannonium; Österreich). In: PIller W. E. (Hrsg.): Stratigraphia Austriaca. Wien: Österreichische Akademie der Wissenschaften (ÖAW), S. 11-62 (= Schriftenreihe der Erdwissenschaftlichen Kommissionen der ÖAW, 16). - https://www.austriaca.at/buecher/files/Schriftenreihe_der Erdwissenschaftlichen_Kommissionen/Stratigraphia\%20Austriaca/02-gross.pdf;internal\& action=opendocument.action (letzter Zugriff: 20.05.2020).

Gruber M., Kanonier A., Pohn-Weidinger S., Schindelegger A. (2018): Raumordnung in Österreich und Bezüge zur Raumentwicklung und Regionalpolitik. Wien: Österreichische 
Raumordnungskonferenz (= ÖROK-Schriftenreihe, 202). - https://www.oerok.gv.at//file admin/user_upload/Bilder/5.Reiter-Publikationen/_\%C3\%96ROK_202_dt._klein_HP.pdf (letzter Zugriff: 20.05.2020).

GüTHLER A. (2008): Kulturlandschaftswandel im Alpenraum. Fallbeispiel südliches Oberallgäu/ Tannheimer Tal. In: Geographische Rundschau, 60 (3), S. 30-36.

HAMPICKe U. (2013): Das Werden der mitteleuropäischen Landschaft und ihr heutiges Bild. In: HAMPICKE U. (Hrsg.): Kulturlandschaft und Naturschutz: Probleme - Konzepte - Ökonomie. Wiesbaden: Springer Vieweg, S. 9-26.

Heiland S., Tischer M., Döring T., Pahl T., Jessel B. (2003): Indikatoren zur Zielkonkretisierung und Erfolgskontrolle im Rahmen der Lokalen Agenda 21. Berlin: Umweltbundesamt. - https://www.umweltbundesamt.de/publikationen/indikatoren-zur-zielkonkretisierung-0 (letzter Zugriff: 20.05.2020).

Hoymann J., Goetzke R. (2014): Die Zukunft der Landnutzung in Deutschland - Darstellung eines methodischen Frameworks. In: Raumforschung und Raumordnung, 72 (3), S. 211-225. https://doi.org/10.1007/s13147-014-0290-y.

Hoymann J., Goetzke R. (2016): Simulation and Evaluation of Urban Growth for Germany Including Climate Change Mitigation and Adaptation Measures. In: ISPRS International Journal of Geo-Information, 5 (7), article 101. - https://doi.org/10.3390/ijgi5070101.

Huang I. B., KeisLer J., Linkov I. (2011): Multi-criteria Decision Analysis in Environmental Sciences: Ten Years of Applications and Trends. In: Science of The Total Environment, 409 (19), S. 3578-3594. - https://doi.org/10.1016/j.scitotenv.2011.06.022.

ILLAS (2020): Integrating Land Use Legacies in Landslide Risk Assessment to Support Spatial Planning - the ILLAS Project. In Zusammenarbeit von AIT (Austrian Institute of Technology $\mathrm{GmbH}$ ), Universität für Bodenkultur Wien, Friedrich-Schiller-Universität Jena und Joanneum Research Forschungsgesellschaft mbH Graz (= Austrian Climate Research Programme 2016, Ausschreibung 9, Klimafonds-Nr: B670294). - https://www.klimafonds.gv.at/wp-content/up loads/sites/6/B670294-ACRP9-ILLAS-KR16AC0K13226-EB.pdf(letzter Zugriff: 07.12.2020).

JAEGer J. (2000): Landscape Division, Splitting Index, and Effective Mesh Size: New Measures of Landscape Fragmentation. In: Landscape Ecology, 15 (2), S. 115-130. - https://doi.org/ 10.1023/A:1008129329289.

Jaeger J., Schwick C., Hennig E. I., Schwarzak M., Krüger T., Behnisch M., Soukup T., Orlitova E., Nazarnia N., Kienast F. (2018): Messung der Zersiedelung ermöglicht Monitoring und stärkere Berücksichtigung in der Planung. In: BeHNisCH M., KRETSCHMER O., MeInel G. (Hrsg.): Flächeninanspruchnahme in Deutschland: Auf dem Weg zu einem besseren Verständnis der Siedlungs- und Verkehrsflächenentwicklung. Berlin: Springer, S. 245-267. - https://doi.org/10.1007/978-3-662-50305-8_14.

KLEIN K. (1973): Die Bevölkerung Österreichs vom Beginn des 16. bis zur Mitte des 18. Jahrhunderts (mit einem Abriss der Bevölkerungsentwicklung von 1754 bis 1869). In: HelCzMANOvszKi H. (Hrsg.): Beiträge zur Bevölkerungs- und Sozialgeschichte Österreichs. Wien: Verlag für Geschichte und Politik, S. 47-112.

KLEIN K. (1990): Die Bevölkerungsentwicklung in den größeren Sammelsiedlungen Österreichs seit dem Hochmittelalter. In: Mitteilungen der Österreichischen Geographischen Gesellschaft, 132 (1), S. 56-90.

KLEINER M. (2006): Planungsinstrumente einer nachhaltigen Raumentwicklung: Indikatorenbasiertes Monitoring und Controlling in der Schweiz, Österreich und Deutschland. Innsbruck: Geographie Innsbruck (= Innsbrucker Geographische Studien, 35).

Knevels R. (2019): VLSM: Vector-based Landscape Metrics. R Package Version 0.0.0-9002. San Francisco: GitHub, Inc. - https://github.com/raff-k/VLSM (letzter Zugriff: 20.05.2020). 
Langthaler E. (2003): Agrarwende in den Bergen. Eine Region in den niederösterreichischen Voralpen (1880-2000). In: Bruckmüller E., Hanisch E., SANDGruber R. (Hrsg.): Geschichte der österreichischen Land-und Forstwirtschaft im 20. Jahrhundert. Regionen - Betriebe Menschen. Wien: Ueberreuter, S. 563-650.

Linser S., Gottsberger T., Peterseil J., Rabitsch W. (2005): Nicht-nachhaltige Trends in Österreich: Qualitative Lebensraumveränderung durch Verlust an biologischer Vielfalt. Wien: Forum Nachhaltiges Österreich / Umweltbundesamt. - http://www.forschungsnetzwerk.at/ downloadpub/Modul3_Qualitatitve_Lebensraumveraenderung.pdf (letzter Zugriff: 20.05. 2020).

LUBW - Landesanstalt für Umwelt, Messungen und Naturschutz Baden-Württemberg (2007): Indikatoren zur Flächeninanspruchnahme und flächensparenden Siedlungsentwicklung. Karlsruhe: LUBW. - https://fachdokumente.lubw.baden-wuerttemberg.de/servlet/ is/20102/indikatoren_zur_flaecheninanspruchnahme.pdf?command=downloadContent $\&$ filename=indikatoren_zur_flaecheninanspruchnahme.pdf\&FIS=199 (letzter Zugriff: 20. 05.2020).

Malczewski J., Rinner C. (2015): Multicriteria Decision Analysis in Geographic Information Science. Berlin: Springer (Advances in Geographic Information Science).

Marcucci D. J. (2000): Landscape History as a Planning Tool. In: Landscape and Urban Planning, 49 (1), S. 67-81. - https://doi.org/10.1016/S0169-2046(00)00054-2.

Moser B., JAeger J., TAPpeiner U., TAsser E., Eiselt B. (2007): Modification of the Effective Mesh Size for Measuring Landscape Fragmentation to Solve the Boundary Problem. In: Landscape Ecology, 22 (3), S. 447-459. - https://doi.org/10.1007/s10980-006-9023-0.

Niedertscheider M., Tasser E., Patek M., Rüdisser J., TAPPeiner U., Erb K.-H. (2017): Influence of Land-Use Intensification on Vegetation C-Stocks in an Alpine Valley from 1865 to 2003. In: Ecosystems, 20 (8), S. 1391-1406. - https://doi.org/10.1007/s10021-017-0120-5.

Olsson E. G. A., Austrheim G., Grenne S. N. (2000): Landscape Change Patterns in Mountains, Land Use and Environmental Diversity, Mid-Norway 1960-1993. In: Landscape Ecology, 15 (2), S. 155-170. - https://doi.org/10.1023/A:1008173628016.

ÖROK - Österreichische Raumordnungskonferenz (2011): Österreichisches Raumentwicklungskonzept (ÖREK) 2011. Wien: ÖROK. - https://www.oerok.gv.at/fileadmin/user_upload/ Bilder/2.Reiter-Raum_u._Region/1.OEREK/OEREK_2011/Dokumente_OEREK_2011/ OEREK_2011_DE_Downloadversion.pdf(letzter Zugriff: 20.05.2020).

Parcerisas L., Marull J., Pino J., Tello E., Coll F., Basnou C. (2012): Land Use Changes, Landscape Ecology and their Socioeconomic Driving Forces in the Spanish Mediterranean Coast (El Maresme County, 1850-2005). In: Environmental Science \& Policy, 23, S. 120-132. https://doi.org/10.1016/j.envsci.2012.08.002.

Plewe B. (2002): The Nature of Uncertainty in Historical Geographic Information. In: Transactions in GIS, 6 (4), S. 431-456. - https://doi.org/10.1111/1467-9671.00121.

PlöGer R. (1999): Anwendung geographischer Informationssysteme in der Angewandten Historischen Geographie. In: JAKOBS K., KLEEFELD K.-D. (Hrsg.): Informationssysteme für die Angewandte Historische Geographie, S. 103-111 (= Aachener Informatik-Berichte, 99-6).

R Core Team (2018): R: A Language and Environment for Statistical Computing. Version 3.5.2. Wien: R Foundation for Statistical Computing. - https://www.R-project.org/ (letzter Zugriff: 20.05.2020).

Reichenbach P., Busca C., Mondini A. C., Rossi M. (2014): The Influence of Land Use Change on Landslide Susceptibility Zonation: The Briga Catchment Test Site (Messina, Italy). In: Environmental Management, 54 (6), S. 1372-1384 - https://doi.org/10.1007/s00267-0140357-0. 
RoEDENBECK I. A. (2005): Entwicklung der Landschaftszerschneidung in Hessen von 1930 bis 2002. Gießen: Institut für Biometrie und Populationsgenetik, Justus-Liebig-Universität Gießen (= Statusbericht für das Hessische Landesamt für Umwelt und Geologie (HLUG)). - http://static.hlug.de/medien/nachhaltigkeit/dokumente/Roedenbeck_BerichtHistorie.pdf\# page $=1$ \&zoom =auto, $-274,848$ (letzter Zugriff: 20.05.2020).

RoEDENBECK I. A., KöHLER W. (2006): Effekte der Landschaftszerschneidung auf die Unfallhäufigkeit und Bestandsdichte von Wildtierpopulationen. In: Naturschutz und Landschaftsplanung, 38 (10-11), S. 314-322.

Salvati L., Zuliani E. D., Sabbi A., Cancellieri L., Tufano M., Caneva G., Savo V. (2017): Land-cover Changes and Sustainable Development in a Rural Cultural Landscape of Central Italy: Classical Trends and Counter-intuitive Results. In: International Journal of Sustainable Development \& World Ecology, 24 (1), S. 27-36. - https://doi.org/10.1080/13504509. 2016.1193778 .

SANDGRUBER R. (1978): Österreichische Agrarstatistik 1750-1918. Wien: Verlag für Geschichte und Politik (Wirtschafts- und Sozialstatistik Österreich-Ungarns).

SCHENK W. (2002): Aktuelle Tendenzen der Landschaftsentwicklung in Deutschland und Aufgaben der Kulturlandschaftspflege. In: Petermanns Geographische Mitteilungen, 146 (6), S. 54-57.

SchenK W. (2016): Kulturlandschaft als gedankliches Konstrukt auf der Basis eines realen Substrats. In: Deutsche Gesellschaft für Gartenkunst und Landschaftskultur (DGGL) (Hrsg.): Landschaftskultur. Zwischen Bewahrung und Entwicklung. München: Callwey, S. 18-21 (= DGGL-Jahrbuch, 11).

Schindler S., Banko G., Moser D., Grillmayer R., Zulka K. P., Lamb U., Essl F., Stejskal-TieFENBACH M. (2017): Österreichisches Biodiversitäts-Monitoring (ÖBM) - Kulturlandschaft. Konzept für die Erfassung von Status und Trends der Biodiversität. Wien: Umweltbundesamt (= Report, 0635). - https://www.umweltbundesamt.at/aktuell/publikationen/publikations suche/publikationsdetail/?pub_id=2262 (letzter Zugriff: 20.05.2020).

Siedentop S., Heiland S., Lehmann I., Hernig A., Schauerte-LüKe N. (2007): Nachhaltigkeitsbarometer Fläche. Regionale Schlüsselindikatoren nachhaltiger Flächennutzung für die Fortschrittsberichte der nationalen Nachhaltigkeitsstrategie - Flächenziele. Bonn: Bundesministerium für Verkehr, Bau und Stadtentwicklung (BMVBS) und Bundesamt für Bauwesen und Raumordnung (BBR) (= Forschungen, 130). - https://www.bbsr.bund.de/BBSR/ DE/Veroeffentlichungen/ministerien/BMVBS/Forschungen/2007/Heft130 (letzter Zugriff: 20.05.2020).

Statistik Austria (2011a): Ein Blick auf die Gemeinde Waidhofen an der Ybbs (30301). Österreich besser verstehen. Bevölkerungsentwicklung 1869-2019. Wien: Bundesanstalt Statistik Austria. - https://www.statistik.at/blickgem/G0201/g30301.pdf(letzter Zugriff: 20.05.2020).

Statistik Austria (2011b): Ein Blick auf die Gemeinde Paldau (62384). Österreich besser verstehen. Bevölkerungsentwicklung 1869-2019. Wien: Bundesanstalt Statistik Austria. - https:// www.statistik.at/blickgem/G0201/g62384.pdf(letzter Zugriff: 20.05.2020).

TAsser E., Ruffini F. V., TAPPeiner U. (2009): An Integrative Approach for Analysing Landscape Dynamics in Diverse Cultivated and Natural Mountain Areas. In: Landscape Ecology, 24 (5), S. 611-628. - https://doi.org/10.1007/s10980-009-9337-9.

Walz U. (2008): Monitoring of Landscape Change and Functions in Saxony (Eastern Germany) - Methods and Indicators. In: Ecological Indicators, 8 (6), S. 807-817. - https://doi. org/10.1016/j.ecolind.2007.09.006.

WALz U. (2013): Landschaftsstrukturmaße und Indikatorensysteme zur Erfassung und Bewertung des Landschaftswandels und seiner Umweltauswirkungen - unter besonderer Berücksichtigung der biologischen Vielfalt. Habilitationsschrift. Rostock: Universität Rostock. - http:// 
rosdok.uni-rostock.de/file/rosdok_disshab_0000000980/rosdok_derivate_0000005089/ Habilitationsschrift_Walz_2013.pdf (letzter Zugriff: 20.05.2020).

Walz U., SteIN C. (2017): Indikatoren für ein räumliches Monitoring des Landschaftswandels. In: WeNDE W., WALz U. (Hrsg.): Die räumliche Wirkung der Landschaftsplanung: Evaluation, Indikatoren und Trends. Wiesbaden: Springer Fachmedien, S. 57-75. - https://doi.org/ 10.1007/978-3-658-13556-0_5.

Weiss P., Schieler K., Schadauer K., Englisch M. (2000): Die Kohlenstoffbilanz des österreichischen Waldes und Betrachtungen zum Kyoto-Protokoll. Wien: Umweltbundesamt (= Monographien, 106). - https://www.umweltbundesamt.at/fileadmin/site/publikationen/M106z.pdf (letzter Zugriff: 20.05.2020).

Wessely G. (2006): Geologie der Österreichischen Bundesländer: Niederösterreich. Wien: Verlag der Geologischen Bundesanstalt.

Wrbka T., Reiter K., PaAr M., Szerencsits E., Stocker-Kiss A., Fussenegger K. (2005): Die Landschaften Österreichs und ihre Bedeutung für die biologische Vielfalt. Wien: Umweltbundesamt (= Monographien, M-173). - https://www.umweltbundesamt.at/fileadmin/site/ publikationen/M173.pdf (letzter Zugriff: 20.05.2020).

\section{Anhang}

\begin{tabular}{|c|c|c|c|c|}
\hline \multirow{2}{*}{ Indikator } & \multicolumn{3}{|c|}{ Paldau (Indexwerte) } & \multirow{2}{*}{$\begin{array}{l}\text { Referenzwert } 1820 \\
\text { (= Index 100) }\end{array}$} \\
\hline & 1820 & 1960 & 2015 & \\
\hline \multicolumn{5}{|c|}{ Siedlungsentwicklung (S) } \\
\hline $\mathrm{S} 1^{*}$ & 100 & 4,95 & 0 & 2,66 \\
\hline S2.1 & 100 & 82,3 & 29,77 & 59,14 \\
\hline $\mathrm{S} 2.2 *$ & 100 & 58,01 & 0 & 2,05 \\
\hline S3 & 100 & 94,65 & 2,41 & $3.626,91$ \\
\hline S4 & 100 & 49,12 & 13,9 & $2.997,85$ \\
\hline \multicolumn{5}{|l|}{ Waldentwicklung (W) } \\
\hline W1 & 100 & 72,79 & 98,04 & 13,8 \\
\hline $\mathrm{W} 2 *$ & 100 & 101,81 & 0 & $2.237,95$ \\
\hline W3 & 100 & 90,04 & 92,02 & 36,17 \\
\hline \multicolumn{5}{|c|}{ Landwirtschaftsentwicklung (L) } \\
\hline L1 & 100 & 38,11 & 7,36 & 0,59 \\
\hline L2* & 100 & 0 & 0 & $1.137,31$ \\
\hline L3 & 100 & 150,09 & 70,9 & 30,17 \\
\hline
\end{tabular}

* Aufsteigende Ursprungswerte sind negativ zu bewerten.

Indikatoren: S1: Flächeninanspruchnahme; S2.1: Freiflächeneffizienz; S2.2: Zerklüftungsgrad; S3: Zerschneidung; S4: Siedlungsdichte; W1: Waldfragmentierung; W2: Waldnutzungsintensität; W3: Waldflächenanteil; L1: Ökotone; L2: Landwirtschaftliche Intensität; L3: Grünlandanteil an der Landwirtschaftsfläche

Tab. A1: Indizes nach Referenzierung von Paldau mit Werten von Paldau 1820 


\begin{tabular}{|c|c|c|c|c|}
\hline \multirow{2}{*}{ Indikator } & \multicolumn{3}{|c|}{ Waidhofen (Indexwerte) } & \multirow{2}{*}{$\begin{array}{l}\text { Referenzwert } 1820 \\
\quad(=\text { Index 100) }\end{array}$} \\
\hline & 1820 & 1960 & 2015 & \\
\hline \multicolumn{5}{|c|}{ Siedlungsentwicklung (S) } \\
\hline $\mathrm{S} 1 *$ & 100 & 81,87 & 0 & 1,87 \\
\hline $\mathrm{S} 2.1$ & 100 & 116,77 & 50,87 & 61,55 \\
\hline $\mathrm{S} 2.2 *$ & 100 & 79,66 & 0 & 2,04 \\
\hline S3 & 100 & 197,74 & 38,33 & $3.220,94$ \\
\hline S4 & 100 & 126,54 & 24,73 & $3.235,42$ \\
\hline \multicolumn{5}{|l|}{ Waldentwicklung (W) } \\
\hline W1 & 100 & 135,54 & 200 & 148,51 \\
\hline $\mathrm{W} 2 *$ & 100 & 105,63 & 0 & $2.328,05$ \\
\hline W3 & 100 & 107,88 & 122,64 & 35,37 \\
\hline \multicolumn{5}{|c|}{ Landwirtschaftsentwicklung (L) } \\
\hline $\mathrm{L} 1$ & 100 & 137,53 & 20,28 & 0,46 \\
\hline $\mathrm{L} 2 *$ & 100 & 0 & 0 & 755,19 \\
\hline L3 & 100 & 152,02 & 168,49 & 58,04 \\
\hline
\end{tabular}

* Aufsteigende Ursprungswerte sind negativ zu bewerten. Erläuterung der Indikatoren: siehe Tab. A1 Tab. A2: Indizes nach Referenzierung von Waidhofen mit Werten von Waidhofen 1820

\begin{tabular}{|c|c|c|c|c|c|c|c|}
\hline \multirow{2}{*}{ Indikator } & \multicolumn{3}{|c|}{ Paldau } & \multicolumn{3}{|c|}{ Waidhofen } & \multirow{2}{*}{$\begin{array}{c}\text { Referenz- } \\
\text { wert }\end{array}$} \\
\hline & 1820 & 1960 & 2015 & 1820 & 1960 & 2015 & \\
\hline \multicolumn{8}{|c|}{ Siedlungsentwicklung (S) } \\
\hline $\mathrm{S} 1 *$ & 162,01 & 125,9 & 0 & 173,21 & 168,36 & 44,08 & 6,99 \\
\hline $\mathrm{S} 2.1$ & 122,30 & 100,66 & 36,41 & 127,28 & 148,62 & 64,75 & 48,36 \\
\hline $\mathrm{S} 2.2 *$ & 143,83 & 120,25 & 0 & 144,34 & 133,02 & 75,91 & 3,66 \\
\hline S3 & 121,09 & 114,61 & 2,92 & 107,53 & 200 & 41,22 & $2.995,28$ \\
\hline S4 & 138,19 & 67,87 & 19,2 & 149,14 & 188,72 & 36,88 & $2.169,44$ \\
\hline \multicolumn{8}{|c|}{ Waldentwicklung (W) } \\
\hline W1 & 10,07 & 7,33 & 9,87 & 108,42 & 146,95 & 200 & 136,98 \\
\hline $\mathrm{W} 2 *$ & 131,82 & 133,05 & 27,36 & 129,07 & 133,07 & 45,63 & $3.282,35$ \\
\hline W3 & 99,13 & 89,26 & 91,22 & 96,93 & 104,58 & 118,87 & 36,49 \\
\hline \multicolumn{8}{|c|}{ Landwirtschaftsentwicklung (L) } \\
\hline L1 & 174,44 & 66,49 & 12,83 & 134,30 & 184,70 & 27,24 & 0,34 \\
\hline $\mathrm{L} 2 *$ & 168,77 & 89,58 & 25,36 & 179,26 & 95,42 & 41,60 & $3.642,07$ \\
\hline $\mathrm{L} 3$ & 53,10 & 79,70 & 37,65 & 102,15 & 155,29 & 172,11 & 56,82 \\
\hline
\end{tabular}

* Aufsteigende Ursprungswerte sind negativ zu bewerten. Erläuterung der Indikatoren: siehe Tab. A1 Tab. A3: Indizes nach Durchschnittswert-Referenzierung beider Gemeinden 